\title{
Swiveled Rényi entropies
}

\author{
Frédéric Dupuis* Mark M. Wilde
}

July 2,2018

\begin{abstract}
This paper introduces "swiveled Rényi entropies" as an alternative to the Rényi entropic quantities put forward in [Berta et al., Phys. Rev. A 91, 022333 (2015)]. What distinguishes the swiveled Rényi entropies from the prior proposal of Berta et al. is that there is an extra degree of freedom: an optimization over unitary rotations with respect to particular fixed bases (swivels). A consequence of this extra degree of freedom is that the swiveled Rényi entropies are ordered, which is an important property of the Rényi family of entropies. The swiveled Rényi entropies are however generally discontinuous at $\alpha=1$ and do not converge to the von Neumann entropy-based measures in the limit as $\alpha \rightarrow 1$, instead bounding them from above and below. Particular variants reduce to known Rényi entropies, such as the Rényi relative entropy or the sandwiched Rényi relative entropy, but also lead to ordered Rényi conditional mutual informations and ordered Rényi generalizations of a relative entropy difference. Refinements of entropy inequalities such as monotonicity of quantum relative entropy and strong subadditivity follow as a consequence of the aforementioned properties of the swiveled Rényi entropies. Due to the lack of convergence at $\alpha=1$, it is unclear whether the swiveled Rényi entropies would be useful in one-shot information theory, so that the present contribution represents partial progress toward this goal.
\end{abstract}

\section{Introduction}

In 1961, Alfred Rényi defined a parametrized family of entropies now bearing his name, by relaxing one of the axioms that singles out the Shannon entropy [Rén61]. This led to both the $\alpha$-Rényi entropy and the $\alpha$-Rényi divergence, defined respectively for a parameter $\alpha \in(0,1) \cup(1, \infty)$ and probability distributions $p$ and $q$ as

$$
\begin{aligned}
H_{\alpha}(p) & \equiv \frac{1}{1-\alpha} \log \sum_{x}[p(x)]^{\alpha}, \\
D_{\alpha}(p \| q) & \equiv \frac{1}{\alpha-1} \log \sum_{x}[p(x)]^{\alpha}[q(x)]^{1-\alpha},
\end{aligned}
$$

\footnotetext{
${ }^{*}$ Faculty of Informatics, Masaryk University, Brno, Czech Republic

${ }^{\dagger}$ Hearne Institute for Theoretical Physics, Department of Physics and Astronomy, Center for Computation and Technology, Louisiana State University, Baton Rouge, Louisiana 70803, USA
} 
where log denotes the natural logarithm here and throughout the paper. The Shannon entropy and relative entropy are recovered in the limit as $\alpha \rightarrow 1$ :

$$
\begin{gathered}
\lim _{\alpha \rightarrow 1} H_{\alpha}(p)=H(p) \equiv-\sum_{x} p(x) \log p(x), \\
\lim _{\alpha \rightarrow 1} D_{\alpha}(p \| q)=D(p \| q) \equiv \sum_{x} p(x) \log \frac{p(x)}{q(x)} .
\end{gathered}
$$

What began largely as a theoretical exploration ended up having many practical ramifications, especially in the contexts of information theory and statistics. For example, it is now well known that the Rényi entropies play a fundamental role in obtaining a sharpened understanding of the trade-off between communication rate, error probability, and number of resources in communication protocols, such as data compression and channel coding [Csi95, vEH14]. "Smoothing" the Rényi entropies [RW04] has also led to the development of "one-shot" information theory [Ren05, Tom12], with applications to cryptography.

Part of what makes the Rényi entropies so useful in applications is their properties: convergence to the Shannon and relative entropies in the limit as $\alpha \rightarrow 1$, monotonicity in the parameter $\alpha$, and additivity, in addition to others. The convergence to the Shannon and relative entropies ensures that, by taking this limit, one recovers asymptotic information-theoretic statements, such as the data compression theorem or the channel capacity theorem, from the more fine-grained statements. Monotonicity in the parameter $\alpha$ ensures that $H_{\alpha}(p)$ gives more weight to low surprisal events for $\alpha>1$ and vice versa for $\alpha<1$, helping to characterize the aforementioned trade-off in informationtheoretic settings. The additivity property implies that the Rényi entropies can simplify immensely when evaluated for memoryless stochastic processes.

In light of the progress that the Rényi paradigm has brought to information theory, one is left to wonder if this could happen in more exotic settings, such as quantum information theory and/or for "multipartite" settings (here by multipartite, we mean three or more parties). This line of thought has led to the development of several non-commutative generalizations of the Rényi relative entropy in (1.2), which has in turn led to a sharpened understanding of several quantum information-theoretic tasks (see [CMW14, Tom15] and references therein) and refinements of the uncertainty principle CBTW15]. As far as we are aware, the development of the multipartite generalization of the Rényi entropy in (1.1) is less explored, with the exception of a recent proposal [BSW15b] for a multipartite quantum generalization.

With the intent of developing either a multipartite classical or quantum generalization of (1.1), one might suggest after a moment's thought to replace a quantity which features a linear combination of entropies by one with the same linear combination of Rényi entropies. However, this approach is objectively unsatisfactory in at least two regards: properties of the original information measure are not preserved by doing so and one is not guaranteed to have the powerful monotonicity in $\alpha$ property mentioned above. For example, take the case of the conditional mutual information of a tripartite density operator $\rho_{A B C}$ defined as

$$
I(A ; B \mid C)_{\rho} \equiv H(A C)_{\rho}+H(B C)_{\rho}-H(C)_{\rho}-H(A B C)_{\rho},
$$

where $H(F)_{\sigma} \equiv-\operatorname{Tr}\left\{\sigma_{F} \log \sigma_{F}\right\}$ is the quantum entropy of a density operator $\sigma$ on system $F$. One of the most important properties of this quantity is that it is non-negative (known as strong subadditivity of quantum entropy [LR73a, LR73b]), and as a consequence, it is monotone nonincreasing with respect to any quantum channel applied to the system $A$ [CW04] (by symmetry, 
the same is true for one applied to $B)$. However, if we define a Rényi generalization of $I(A ; B \mid C)_{\rho}$ as $H_{\alpha}(A C)_{\rho}+H_{\alpha}(B C)_{\rho}-H_{\alpha}(C)_{\rho}-H_{\alpha}(A B C)_{\rho}$, where $H_{\alpha}(F)_{\sigma} \equiv\left[\log \operatorname{Tr}\left\{\sigma_{F}^{\alpha}\right\}\right] /(1-\alpha)$, then explicit counterexamples reveal that this Rényi generalization can be negative, monotonicity with respect to quantum channels need not hold, and neither does monotonicity in $\alpha$ [LMW13].

To remedy these deficiencies, the authors of [BSW15b] put forward a general prescription for producing a Rényi generalization of a quantum information measure, with the aim of having the properties of the original measure retained while also satisfying the monotonicity in $\alpha$ property. The work in [BSW15b] was only partially successful in this regard. Continuing with our example of conditional mutual information, consider the following Rényi generalization [BSW15a]:

$$
I_{\alpha}(A ; B \mid C)_{\rho} \equiv \frac{1}{\alpha-1} \log \operatorname{Tr}\left\{\rho_{A B C}^{\alpha} \rho_{A C}^{(1-\alpha) / 2} \rho_{C}^{(\alpha-1) / 2} \rho_{B C}^{1-\alpha} \rho_{C}^{(\alpha-1) / 2} \rho_{A C}^{(1-\alpha) / 2}\right\} .
$$

For $\alpha \in[0,1) \cup(1,2]$, the quantity is non-negative, monotone non-increasing with respect to quantum channels acting on the $B$ system, converges to $I(A ; B \mid C)_{\rho}$ in the limit as $\alpha \rightarrow 1$, and is conjectured to obey the monotonicity in $\alpha$ property (with some numerical and analytical evidence in favor established) [BSW15a]. However, hitherto a proof of the monotonicity in $\alpha$ property for $I_{\alpha}(A ; B \mid C)_{\rho}$ remains lacking. It is also an open question to determine whether $I_{\alpha}(A ; B \mid C)_{\rho}$ is monotone non-increasing with respect to quantum channels acting on the $A$ system - this partially has to do with the fact that $I_{\alpha}(A ; B \mid C)_{\rho}$ is not symmetric with respect to exchange of the $A$ and $B$ systems, unlike the conditional mutual information in (1.5).

\section{Summary of results}

In this paper, we modify the recently proposed Rényi generalizations of quantum information measures from [BSW15b] by placing "swivels" in a given chain of operators 1 As an example of the idea, consider that we can rewrite the quantity in (1.6) in terms of the Schatten 2-norm as follows:

$$
I_{\alpha}(A ; B \mid C)_{\rho} \equiv \frac{2}{\alpha-1} \log \left\|\rho_{B C}^{(1-\alpha) / 2} \rho_{C}^{(\alpha-1) / 2} \rho_{A C}^{(1-\alpha) / 2} \rho_{A B C}^{\alpha / 2}\right\|_{2} .
$$

The new idea is to modify this quantity to include swivels as follows:

$$
I_{\alpha}^{\prime}(A ; B \mid C)_{\rho} \equiv \frac{2}{\alpha-1} \max _{V_{\rho_{A C}} \in \mathbb{V}_{\rho_{A C}}, V_{\rho_{C}} \in \mathbb{V}_{\rho_{C}}} \log \left\|\rho_{B C}^{(1-\alpha) / 2} V_{\rho_{C}} \rho_{C}^{(\alpha-1) / 2} \rho_{A C}^{(1-\alpha) / 2} V_{\rho_{A C}} \rho_{A B C}^{\alpha / 2}\right\|_{2},
$$

where $\mathbb{V}_{\omega}$ is the compact set of all unitaries $V_{\omega}$ commuting with the Hermitian operator $\omega$. Thus, the fixed eigenbases of $\rho_{C}$ and $\rho_{A C}$ act as swivels connecting adjacent operators in the operator chain above, such that the unitary rotations $V_{\rho_{C}}$ and $V_{\rho_{A C}}$ about these swivels are allowed. Of course, such swivels make no difference when the density operator $\rho_{A B C}$ and its marginals commute with each other (the classical case), or when the $C$ system is trivial, in which case the above quantity reduces to a Rényi mutual information

$$
\begin{aligned}
I_{\alpha}^{\prime}(A ; B)_{\rho} & \equiv \frac{2}{\alpha-1} \max _{V_{\rho_{A}} \in \mathbb{V}_{\rho_{A}}} \log \left\|\rho_{B}^{(1-\alpha) / 2} \rho_{A}^{(1-\alpha) / 2} V_{\rho_{A}} \rho_{A B}^{\alpha / 2}\right\|_{2} \\
& =\frac{2}{\alpha-1} \log \left\|\rho_{B}^{(1-\alpha) / 2} \rho_{A}^{(1-\alpha) / 2} \rho_{A B}^{\alpha / 2}\right\|_{2} .
\end{aligned}
$$

\footnotetext{
${ }^{1} \mathrm{~A}$ "swivel" is a coupling placed between two objects in a chain in order to allow for them to "swivel" about a given axis.
} 
We mention that we were led to the definition in (2.2) as a consequence of the developments in Wil15, in which similar swivels appeared in refinements of entropy inequalities such as monotonicity of quantum relative entropy and strong subadditivity.

The quantity in (2.2) satisfies some of the properties already established for $I_{\alpha}(A ; B \mid C)_{\rho}$ in BSW15a, which include non-negativity for $\alpha \in[0,1) \cup(1,2]$ and monotonicity with respect to quantum channels acting on the $B$ system. However, the extra degree of freedom in (2.2) allows us to prove that this swiveled Rényi conditional mutual information is monotone non-decreasing in $\alpha$ for $\alpha \in[0,1) \cup(1,2]$.

The swiveled Rényi entropies are in general discontinuous at $\alpha=1$ and do not converge to the von Neumann entropy-based measures in the limit as $\alpha \rightarrow 1$. Thus, the present paper represents a work in progress toward the general goal of find Rényi generalizations of quantum information measures that satisfy all of the desired properties that one would like to have. It thus remains an open question to find Rényi quantities that meet all desiderata.

The rest of the paper proceeds by developing this idea in detail. We review some background material in Section 3, which includes various quantum Rényi entropies and the Hadamard three-line theorem, the latter being the essential tool for establishing monotonicity in $\alpha$ for the swiveled Rényi entropies. We then focus in Section 4 on developing swiveled Rényi generalizations of the quantum relative entropy difference in (3.3), given that many different information measures can be written in terms of this relative entropy difference, including conditional mutual information (see, e.g., the discussions in [SBW15, Wil14, Wil15]). Our main contributions are Theorems 6] and 7, which state that these quantities are monotone non-decreasing in $\alpha$ for particular values. We then briefly discuss how refinements of entropy inequalities follow as a consequence of the properties of the swiveled Rényi entropies. Section 5 discusses swiveled Rényi conditional mutual informations and justifies that they possess the properties stated above. We extend the idea in Section 6 to establish swiveled Rényi generalizations of an arbitrary linear combination of von Neumann entropies with coefficients chosen from the set $\{-1,0,1\}$. We finally show how our methods can be used to address an open question posed in [Zha14]. Section 8 concludes with a summary and some open directions.

\section{Preliminaries}

\subsection{Quantum states and channels}

A quantum state is described mathematically by a density operator, which is a positive semi-definite operator with trace equal to one. A quantum channel is a linear, trace-preserving, completely positive map. For more background on quantum information theory, we refer to [NC10, Wil13].

Our results apply to finite-dimensional Hilbert spaces. For most developments, we take $\rho, \sigma$, and $\mathcal{N}$ to be as given in the following definition:

Definition 1 Let $\rho$ be a density operator acting on a finite-dimensional Hilbert space $\mathcal{H}, \sigma$ be a non-zero positive semi-definite operator acting on $\mathcal{H}$, and $\mathcal{N}$ be a quantum channel, taking operators acting on $\mathcal{H}$ to those acting on a finite-dimensional Hilbert space $\mathcal{K}$.

Sometimes we need more restrictions, in which case we take $\rho, \sigma$, and $\mathcal{N}$ as follows:

Definition 2 Let $\rho, \sigma$, and $\mathcal{N}$ be as given in Definition 1 , with the additional restriction that $\rho$ and $\sigma$ are positive definite, and $\mathcal{N}$ is such that $\mathcal{N}(\rho)$ and $\mathcal{N}(\sigma)$ are also positive definite. 
We employ the common convention that functions of Hermitian operators are evaluated on their support. In more detail, the support of a Hermitian operator $A$, written as $\operatorname{supp}(A)$, is defined as the vector space spanned by its eigenvectors whose corresponding eigenvalues are nonzero. Let an eigendecomposition of $A$ be given as $A=\sum_{i: a_{i} \neq 0} a_{i}|i\rangle\langle i|$ for eigenvectors $\{|i\rangle\}$. Then $\operatorname{supp}(A)=\operatorname{span}\left\{|i\rangle: a_{i} \neq 0\right\}$. Let $\Pi_{A}$ denote the projection onto the support of $A$. A function $f$ of an operator $A$ is then defined as $f(A)=\sum_{i: a_{i} \neq 0} f\left(a_{i}\right)|i\rangle\langle i|$.

\subsection{Entropies and norms}

Let $\rho, \sigma$, and $\mathcal{N}$ be as given in Definition 1, The quantum relative entropy [Ume62] is defined as

$$
D(\rho \| \sigma) \equiv \operatorname{Tr}\{\rho[\log \rho-\log \sigma]\},
$$

whenever $\operatorname{supp}(\rho) \subseteq \operatorname{supp}(\sigma)$, and otherwise, it is defined to be equal to $+\infty$. The quantum relative entropy is monotone non-increasing with respect to quantum channels [Lin75, Uhl77, in the sense that

$$
D(\rho \| \sigma) \geq D(\mathcal{N}(\rho) \| \mathcal{N}(\sigma)) .
$$

Another relevant information measure is the quantum relative entropy difference, defined as

$$
\Delta(\rho, \sigma, \mathcal{N}) \equiv D(\rho \| \sigma)-D(\mathcal{N}(\rho) \| \mathcal{N}(\sigma)) .
$$

We can use the Schatten norms in order to establish Rényi generalizations of von Neumann entropies, which are more refined information measures for quantum states and channels that reduce to the von Neumann quantities in a limit. The Schatten $p$-norm of an operator $A$ is defined as

$$
\|A\|_{p} \equiv\left[\operatorname{Tr}\left\{|A|^{p}\right\}\right]^{1 / p}
$$

where $p \geq 1$ and $|A| \equiv \sqrt{A^{\dagger} A}$ (note that we sometimes use the notation $\|A\|_{p}$ even for values $p \in(0,1)$ when the quantity on the right-hand side of (3.4) is not a norm). From the above definition, we can see that the following equalities hold for any operators $A$ and $B$ :

$$
\begin{aligned}
\operatorname{Tr}\left\{B^{\dagger} A^{\dagger} A B\right\} & =\|A B\|_{2}^{2}, \\
\left\|B^{\dagger} A^{\dagger} A B\right\|_{p}^{p} & =\|A B\|_{2 p}^{2 p} .
\end{aligned}
$$

The quantum Rényi entropy of a state $\rho$ is defined for $\alpha \in(0,1) \cup(1, \infty)$ as

$$
H_{\alpha}(\rho) \equiv \frac{1}{1-\alpha} \log \operatorname{Tr}\left\{\rho^{\alpha}\right\}=\frac{\alpha}{1-\alpha} \log \|\rho\|_{\alpha},
$$

and reduces to the von Neumann entropy in the limit as $\alpha \rightarrow 1$ :

$$
\lim _{\alpha \rightarrow 1} H_{\alpha}(\rho)=H(\rho)
$$

There are at least two ways to generalize the quantum relative entropy, which we refer to as the Rényi relative entropy $D_{\alpha}(\rho \| \sigma)$ [Pet86a] and the sandwiched Rényi relative entropy $\widetilde{D}_{\alpha}(\rho \| \sigma)$ 
MLDS $^{+} 13$, WWY14]. They are defined respectively as follows:

$$
\begin{aligned}
D_{\alpha}(\rho \| \sigma) & \equiv \frac{1}{\alpha-1} \log \operatorname{Tr}\left\{\rho^{\alpha} \sigma^{1-\alpha}\right\} \\
& =\frac{2}{\alpha-1} \log \left\|\sigma^{(1-\alpha) / 2} \rho^{\alpha / 2}\right\|_{2} \\
\widetilde{D}_{\alpha}(\rho \| \sigma) & \equiv \frac{1}{\alpha-1} \log \operatorname{Tr}\left\{\left(\sigma^{(1-\alpha) / 2 \alpha} \rho \sigma^{(1-\alpha) / 2 \alpha}\right)^{\alpha}\right\} \\
& =\frac{2 \alpha}{\alpha-1} \log \left\|\sigma^{(1-\alpha) / 2 \alpha} \rho^{1 / 2}\right\|_{2 \alpha},
\end{aligned}
$$

if $\alpha \in(0,1)$ or if $\alpha \in(1, \infty)$ and $\operatorname{supp}(\rho) \subseteq \operatorname{supp}(\sigma)$. If $\alpha \in(1, \infty)$ and $\operatorname{supp}(\rho) \nsubseteq \operatorname{supp}(\sigma)$, then they are defined to be equal to $+\infty$. The rewritings in (3.10) and (3.12) are helpful for our developments in this paper and follow from (3.5)-(3.6) and the following:

$$
\begin{aligned}
\operatorname{Tr}\left\{\rho^{\alpha} \sigma^{1-\alpha}\right\} & =\operatorname{Tr}\left\{\rho^{\alpha / 2} \sigma^{(1-\alpha) / 2} \sigma^{(1-\alpha) / 2} \rho^{\alpha / 2}\right\} \\
\operatorname{Tr}\left\{\left(\sigma^{(1-\alpha) / 2 \alpha} \rho \sigma_{\alpha}^{(1-\alpha) / 2 \alpha}\right)^{\alpha}\right\} & =\left\|\sigma^{(1-\alpha) / 2 \alpha} \rho \sigma^{(1-\alpha) / 2 \alpha}\right\|_{\alpha}^{\alpha} \\
& =\left\|\rho^{1 / 2} \sigma^{(1-\alpha) / \alpha} \rho^{1 / 2}\right\|_{\alpha}^{\alpha} .
\end{aligned}
$$

Both Rényi generalizations reduce to the quantum relative entropy in the limit as $\alpha \rightarrow 1$ [Pet86a, MLDS $^{+} 13$, WWY14:

$$
\lim _{\alpha \rightarrow 1} D_{\alpha}(\rho \| \sigma)=\lim _{\alpha \rightarrow 1} \widetilde{D}_{\alpha}(\rho \| \sigma)=D(\rho \| \sigma)
$$

The Rényi relative entropy is monotone non-increasing with respect to quantum channels when $\alpha \in[0,1) \cup(1,2]$ Pet86a]:

$$
D_{\alpha}(\rho \| \sigma) \geq D_{\alpha}(\mathcal{N}(\rho) \| \mathcal{N}(\sigma)),
$$

and the sandwiched Rényi relative entropy possesses a similar monotonicity property when $\alpha \in$ $[1 / 2,1) \cup(1, \infty)$ [FL13, Bei13]:

$$
\widetilde{D}_{\alpha}(\rho \| \sigma) \geq \widetilde{D}_{\alpha}(\mathcal{N}(\rho) \| \mathcal{N}(\sigma)) .
$$

By picking particular values of the Rényi parameter $\alpha$, the quantities above take on special forms and have meaning in operational contexts, being known as the zero-relative entropy [Dat09], the collision relative entropy [DFW15], the min-relative entropy [ $\mathrm{DKF}^{+} 12$, and the max-relative entropy [Dat09], respectively:

$$
\begin{aligned}
D_{0}(\rho \| \sigma) & =-\log \operatorname{Tr}\left\{\rho^{0} \sigma\right\} \\
D_{2}(\rho \| \sigma) & =\log \left\|\rho \sigma^{-1 / 2}\right\|_{2} \\
\widetilde{D}_{1 / 2}(\rho \| \sigma) & =-\log F(\rho, \sigma) \\
D_{\max }(\rho \| \sigma) & =\lim _{\alpha \rightarrow \infty} \widetilde{D}_{\alpha}(\rho \| \sigma)=\log \left\|\sigma^{-1 / 2} \rho \sigma^{-1 / 2}\right\|_{\infty}=\log \left\|\sigma^{-1 / 2} \rho^{1 / 2}\right\|_{\infty}^{2}
\end{aligned}
$$

where $F(\rho, \sigma) \equiv\|\sqrt{\rho} \sqrt{\sigma}\|_{1}^{2}$ is the quantum fidelity Uhl76]. 


\subsection{Hadamard three-line theorem}

One of the most important technical tools for proving our main result is the operator version of the Hadamard three-line theorem given in [Bei13], in particular, the very slight modification stated in Dup15. We note that the theorem below is a variant of the Riesz-Thorin operator interpolation theorem (see, e.g., [BL76, RS75]).

Theorem 3 Let $S \equiv\{z \in \mathbb{C}: 0 \leq \operatorname{Re}\{z\} \leq 1\}$, and let $L(\mathcal{H})$ be the space of bounded linear operators acting on a Hilbert space $\mathcal{H}$. Let $G: S \rightarrow L(\mathcal{H})$ be a bounded map that is holomorphic on the interior of $S$ and continuous on the boundary 2 Let $\theta \in(0,1)$ and define $p_{\theta}$ by

$$
\frac{1}{p_{\theta}}=\frac{1-\theta}{p_{0}}+\frac{\theta}{p_{1}}
$$

where $p_{0}, p_{1} \in[1, \infty]$. For $k=0,1$ define

$$
M_{k}=\sup _{t \in \mathbb{R}}\|G(k+i t)\|_{p_{k}} .
$$

Then

$$
\|G(\theta)\|_{p_{\theta}} \leq M_{0}^{1-\theta} M_{1}^{\theta}
$$

\subsection{Rényi generalizations of the quantum relative entropy difference}

Let $\rho, \sigma$, and $\mathcal{N}$ be as given in Definition 1. In [SBW15], two Rényi generalizations of the relative entropy difference in (3.3) were defined as follows:

$$
\begin{aligned}
& \Delta_{\alpha}(\rho, \sigma, \mathcal{N}) \equiv \frac{1}{\alpha-1} \log \operatorname{Tr}\left\{\rho^{\alpha} \sigma^{(1-\alpha) / 2} \mathcal{N}^{\dagger}\left([\mathcal{N}(\sigma)]^{(\alpha-1) / 2}[\mathcal{N}(\rho)]^{1-\alpha}[\mathcal{N}(\sigma)]^{(\alpha-1) / 2}\right) \sigma^{(1-\alpha) / 2}\right\} \\
& \widetilde{\Delta}_{\alpha}(\rho, \sigma, \mathcal{N}) \equiv \frac{1}{\alpha^{\prime}} \log \left\|\rho^{1 / 2} \sigma^{-\alpha^{\prime} / 2} \mathcal{N}^{\dagger}\left([\mathcal{N}(\sigma)]^{\alpha^{\prime} / 2}[\mathcal{N}(\rho)]^{-\alpha^{\prime}}[\mathcal{N}(\sigma)]^{\alpha^{\prime} / 2}\right) \sigma^{-\alpha^{\prime} / 2} \rho^{1 / 2}\right\|_{\alpha},
\end{aligned}
$$

where $\alpha^{\prime} \equiv(\alpha-1) / \alpha$. Let $U$ be an isometric extension of $\mathcal{N}$, so that

$$
\mathcal{N}(\cdot)=\operatorname{Tr}_{E}\left\{U(\cdot) U^{\dagger}\right\}
$$

We can write the adjoint $\mathcal{N}^{\dagger}$ in terms of this isometric extension as follows:

$$
\mathcal{N}^{\dagger}(\cdot)=U^{\dagger}\left((\cdot) \otimes I_{E}\right) U
$$

This then allows us to write the definitions above in a simpler form:

$$
\begin{aligned}
& \Delta_{\alpha}(\rho, \sigma, \mathcal{N})=\frac{2}{\alpha-1} \log \left\|\left([\mathcal{N}(\rho)]^{(1-\alpha) / 2}[\mathcal{N}(\sigma)]^{(\alpha-1) / 2} \otimes I_{E}\right) U \sigma^{(1-\alpha) / 2} \rho^{\alpha / 2}\right\|_{2}, \\
& \widetilde{\Delta}_{\alpha}(\rho, \sigma, \mathcal{N})=\frac{2}{\alpha^{\prime}} \log \left\|\left([\mathcal{N}(\rho)]^{-\alpha^{\prime} / 2}[\mathcal{N}(\sigma)]^{\alpha^{\prime} / 2} \otimes I_{E}\right) U \sigma^{-\alpha^{\prime} / 2} \rho^{1 / 2}\right\|_{2 \alpha} .
\end{aligned}
$$

It is known that the following limits hold for $\rho, \sigma$, and $\mathcal{N}$ taken as in Definition 2 [SBW15]:

$$
\lim _{\alpha \rightarrow 1} \Delta_{\alpha}(\rho, \sigma, \mathcal{N})=\lim _{\alpha \rightarrow 1} \widetilde{\Delta}_{\alpha}(\rho, \sigma, \mathcal{N})=\Delta(\rho, \sigma, \mathcal{N})
$$

\footnotetext{
${ }^{2}$ A map $G: S \rightarrow L(\mathcal{H})$ is holomorphic (continuous, bounded) if the corresponding functions to matrix entries are holomorphic (continuous, bounded).
} 
The fact that these limits hold for $\rho, \sigma$, and $\mathcal{N}$ taken as in Definition 1 and subject to $\operatorname{supp}(\rho) \subseteq$ $\operatorname{supp}(\sigma)$ follows from [Wil15] and the development in Appendix A. DW15] proved that for $\alpha \in$ $[0,1) \cup(1,2]$

$$
\Delta_{\alpha}(\rho, \sigma, \mathcal{N}) \geq 0
$$

and for $\alpha \in[1 / 2,1) \cup(1, \infty]$ :

$$
\widetilde{\Delta}_{\alpha}(\rho, \sigma, \mathcal{N}) \geq 0
$$

when $\rho, \sigma$, and $\mathcal{N}$ are taken as in Definition 2, The latter inequality was refined recently in Wil15] for $\alpha \in(1 / 2,1]$ and for $\rho, \sigma$, and $\mathcal{N}$ taken as in Definition 1 and subject to $\operatorname{supp}(\rho) \subseteq \operatorname{supp}(\sigma)$. It remains an open question to determine whether these quantities are non-decreasing in $\alpha$ for any non-trivial range of $\alpha$ (note that SBW15] argued that they are non-decreasing in $\alpha$ in a neighborhood of $\alpha=1$ ).

\section{Swiveled Rényi generalizations of the quantum relative entropy difference}

In the spirit of the discussion in Section 2, we consider different definitions of $\Delta_{\alpha}(\rho, \sigma, \mathcal{N})$ and $\widetilde{\Delta}_{\alpha}(\rho, \sigma, \mathcal{N})$ in order to allow for unitary rotations about swivels, i.e., an optimization over unitaries of the form $V_{\mathcal{N}(\sigma)}$ and $V_{\sigma}$ :

Definition 4 Let $\rho, \sigma$, and $\mathcal{N}$ be as given in Definition 1, We define swiveled Rényi generalizations of the quantum relative entropy difference in (3.3) as follows:

$$
\begin{aligned}
& \Delta_{\alpha}^{\prime}(\rho, \sigma, \mathcal{N}) \equiv \frac{2}{\alpha-1} \max _{V_{\sigma}, V_{\mathcal{N}(\sigma)}} \log \left\|\left([\mathcal{N}(\rho)]^{(1-\alpha) / 2} V_{\mathcal{N}(\sigma)}[\mathcal{N}(\sigma)]^{(\alpha-1) / 2} \otimes I_{E}\right) U \sigma^{(1-\alpha) / 2} V_{\sigma} \rho^{\alpha / 2}\right\|_{2}, \\
& \widetilde{\Delta}_{\alpha}^{\prime}(\rho, \sigma, \mathcal{N}) \equiv \frac{2}{\alpha^{\prime}} \max _{V_{\sigma}, V_{\mathcal{N}(\sigma)}} \log \left\|\left([\mathcal{N}(\rho)]^{-\alpha^{\prime} / 2} V_{\mathcal{N}(\sigma)}[\mathcal{N}(\sigma)]^{\alpha^{\prime} / 2} \otimes I_{E}\right) U \sigma^{-\alpha^{\prime} / 2} V_{\sigma} \rho^{1 / 2}\right\|_{2 \alpha},
\end{aligned}
$$

where $\alpha^{\prime}=(\alpha-1) / \alpha$ and the optimizations are over the compact sets of unitaries $V_{\sigma}$ and $V_{\mathcal{N}(\sigma)}$ commuting with $\sigma$ and $\mathcal{N}(\sigma)$, respectively.

This slight extra degree of freedom allows us to establish that $\Delta_{\alpha}^{\prime}$ and $\widetilde{\Delta}_{\alpha}^{\prime}$ are monotone nondecreasing in $\alpha$ for particular values (see Theorems [6 and 7).

\subsection{Reduction to Rényi relative entropy}

Observe that by choosing $\mathcal{N}=\operatorname{Tr}$, we find that $\Delta_{\alpha}^{\prime}$ reduces to the Rényi relative entropy whenever $\operatorname{supp}(\rho) \subseteq \operatorname{supp}(\sigma)$ :

$$
\begin{aligned}
\Delta_{\alpha}^{\prime}(\rho, \sigma, \operatorname{Tr}) & =\frac{2}{\alpha-1} \log \left\|\sigma^{(1-\alpha) / 2} \rho^{\alpha / 2}\right\|_{2}+\log \operatorname{Tr}\{\sigma\} \\
& =D_{\alpha}(\rho \| \sigma)+\log \operatorname{Tr}\{\sigma\}
\end{aligned}
$$

and $\widetilde{\Delta}_{\alpha}^{\prime}$ to the sandwiched Rényi relative entropy whenever $\operatorname{supp}(\rho) \subseteq \operatorname{supp}(\sigma)$ :

$$
\begin{aligned}
\widetilde{\Delta}_{\alpha}^{\prime}(\rho, \sigma, \operatorname{Tr}) & \equiv \frac{2}{\alpha^{\prime}} \log \left\|\sigma^{-\alpha^{\prime} / 2} \rho^{1 / 2}\right\|_{2 \alpha}+\log \operatorname{Tr}\{\sigma\} \\
& =\widetilde{D}_{\alpha}(\rho \| \sigma)+\log \operatorname{Tr}\{\sigma\}
\end{aligned}
$$


just as

$$
\Delta(\rho, \sigma, \operatorname{Tr})=D(\rho \| \sigma)+\log \operatorname{Tr}\{\sigma\} .
$$

\subsection{Behavior around $\alpha=1$}

Here we discuss the behavior of $\Delta_{\alpha}^{\prime}$ and $\widetilde{\Delta}_{\alpha}^{\prime}$ around $\alpha=1$, with the result being that these quantities are generally discontinuous at $\alpha=1$ :

Proposition 5 Let $\rho, \sigma$, and $\mathcal{N}$ be as given in Definition 2 . Then

$$
\begin{aligned}
& \lim _{\alpha \nearrow 1} \Delta_{\alpha}^{\prime}(\rho, \sigma, \mathcal{N})=\lim _{\alpha \nearrow 1} \widetilde{\Delta}_{\alpha}^{\prime}(\rho, \sigma, \mathcal{N})=\min _{V_{\mathcal{N}(\sigma),}, V_{\sigma}} f\left(1, V_{\mathcal{N}(\sigma)}, V_{\sigma}\right), \\
& \lim _{\alpha \searrow 1} \Delta_{\alpha}^{\prime}(\rho, \sigma, \mathcal{N})=\lim _{\alpha \searrow 1} \widetilde{\Delta}_{\alpha}^{\prime}(\rho, \sigma, \mathcal{N})=\max _{V_{\mathcal{N}(\sigma)}, V_{\sigma}} f\left(1, V_{\mathcal{N}(\sigma)}, V_{\sigma}\right),
\end{aligned}
$$

where

$$
\begin{aligned}
f\left(1, V_{\mathcal{N}(\sigma)}, V_{\sigma}\right) \equiv \operatorname{Tr}\{\rho[\log \rho-\log \sigma]\} & \\
& -\operatorname{Tr}\left\{\mathcal{N}\left(\left[V_{\sigma} \rho V_{\sigma}^{\dagger}\right]\right)\left[\log \left[V_{\mathcal{N}(\sigma)}^{\dagger} \mathcal{N}(\rho) V_{\mathcal{N}(\sigma)}\right]-\log [\mathcal{N}(\sigma)]\right]\right\} .
\end{aligned}
$$

As a consequence, we have that

$$
\min _{V_{\mathcal{N}(\sigma)}, V_{\sigma}} f\left(1, V_{\mathcal{N}(\sigma)}, V_{\sigma}\right) \leq f(1, I, I)=\Delta(\rho, \sigma, \mathcal{N}) \leq \max _{V_{\mathcal{N}(\sigma)}, V_{\sigma}} f\left(1, V_{\mathcal{N}(\sigma)}, V_{\sigma}\right),
$$

and there is generally a discontinuity at $\alpha=1$.

Proof. Let $\mathcal{A} \subseteq[0,2]$, which we will choose shortly. Define the function $f: \mathcal{A} \times \mathbb{V}_{\mathcal{N}(\sigma)} \times \mathbb{V}_{\sigma} \rightarrow \mathbb{R}$ as

$$
f\left(\alpha, V_{\mathcal{N}(\sigma)}, V_{\sigma}\right) \equiv \frac{2}{\alpha-1} \log \left\|\left([\mathcal{N}(\rho)]^{(1-\alpha) / 2} V_{\mathcal{N}(\sigma)}[\mathcal{N}(\sigma)]^{(\alpha-1) / 2} \otimes I_{E}\right) U \sigma^{(1-\alpha) / 2} V_{\sigma} \rho^{\alpha / 2}\right\|_{2},
$$

whenever $\alpha \neq 1$, and $f\left(1, V_{\mathcal{N}(\sigma)}, V_{\sigma}\right)$ as in (4.10). One can check that

$$
\lim _{\alpha \rightarrow 1} f\left(\alpha, V_{\mathcal{N}(\sigma)}, V_{\sigma}\right)=f\left(1, V_{\mathcal{N}(\sigma)}, V_{\sigma}\right),
$$

for example by performing Taylor expansions to calculate the limit (see Appendix C for details of this calculation). The function $f$ is then continuous in $\alpha, V_{\sigma}$, and $V_{\mathcal{N}(\sigma)}$. Furthermore, it fulfills the conditions of Lemma 22 in Appendix B if we choose $\mathcal{A}=[1, M]$ for any $M \in(1,2]$ and $\mathcal{T}=\mathbb{V}_{\mathcal{N}(\sigma)} \times \mathbb{V}_{\sigma}$. Hence, we get that

$$
\Delta_{\alpha}^{\prime}(\rho, \sigma, \mathcal{N})=\max _{V_{\mathcal{N}(\sigma)}, V_{\sigma}} f\left(\alpha, V_{\mathcal{N}(\sigma)}, V_{\sigma}\right)
$$

is continuous on $\alpha \in[1, M]$ and thus

$$
\lim _{\alpha \searrow 1} \Delta_{\alpha}^{\prime}(\rho, \sigma, \mathcal{N})=\max _{V_{\mathcal{N}(\sigma)}, V_{\sigma}} f\left(1, V_{\mathcal{N}(\sigma)}, V_{\sigma}\right)
$$


Repeating the same argument with $\mathcal{A}=[0,1]$ yields that

$$
\Delta_{\alpha}^{\prime}(\rho, \sigma, \mathcal{N})=\min _{V_{\mathcal{N}(\sigma)}, V_{\sigma}} f\left(\alpha, V_{\mathcal{N}(\sigma)}, V_{\sigma}\right)
$$

is continuous on $[0,1]$ and thus

$$
\lim _{\alpha \nearrow 1} \Delta_{\alpha}^{\prime}(\rho, \sigma, \mathcal{N})=\min _{V_{\mathcal{N}(\sigma)}, V_{\sigma}} f\left(1, V_{\mathcal{N}(\sigma)}, V_{\sigma}\right) .
$$

Given that $\Delta(\rho, \sigma, \mathcal{N})=f(1, I, I)$, we can conclude the following inequality:

$$
\min _{V_{\mathcal{N}(\sigma)}, V_{\sigma}} f\left(1, V_{\mathcal{N}(\sigma)}, V_{\sigma}\right) \leq \Delta(\rho, \sigma, \mathcal{N}) \leq \max _{V_{\mathcal{N}(\sigma)}, V_{\sigma}} f\left(1, V_{\mathcal{N}(\sigma)}, V_{\sigma}\right)
$$

The arguments for the quantity $\widetilde{\Delta}_{\alpha}^{\prime}(\rho, \sigma, \mathcal{N})$ are similar, so we just sketch them briefly. Define the function

$$
g\left(\alpha, V_{\mathcal{N}(\sigma)}, V_{\sigma}\right) \equiv \frac{2 \alpha}{\alpha-1} \log \left\|\left([\mathcal{N}(\rho)]^{(1-\alpha) / 2 \alpha} V_{\mathcal{N}(\sigma)}[\mathcal{N}(\sigma)]^{(\alpha-1) / 2 \alpha} \otimes I_{E}\right) U \sigma^{(1-\alpha) / 2 \alpha} V_{\sigma} \rho^{1 / 2}\right\|_{2 \alpha},
$$

for $\alpha \neq 1$ and set $g\left(1, V_{\mathcal{N}(\sigma)}, V_{\sigma}\right)=f\left(1, V_{\mathcal{N}(\sigma)}, V_{\sigma}\right)$. One can then compute (again via Taylor expansions, e.g.) that

$$
\lim _{\alpha \rightarrow 1} g\left(\alpha, V_{\mathcal{N}(\sigma)}, V_{\sigma}\right)=g\left(1, V_{\mathcal{N}(\sigma)}, V_{\sigma}\right) .
$$

The rest of the argument proceeds as above, which leads to the other equalities in (4.8)-(4.9).

\subsection{Monotonicity in the Rényi parameter}

This section contains our main result, that both $\Delta_{\alpha}^{\prime}$ and $\widetilde{\Delta}_{\alpha}^{\prime}$ are monotone non-decreasing with respect to $\alpha$ for particular values.

Theorem 6 Let $\rho, \sigma$, and $\mathcal{N}$ be as given in Definition 1 . The swiveled Rényi quantity $\Delta_{\alpha}^{\prime}(\rho, \sigma, \mathcal{N})$ is monotone non-decreasing with respect to $\alpha \in[0,1) \cup(1,2]$, in the sense that for $0 \leq \alpha \leq \gamma \leq 2$, $\alpha \neq 1$, and $\gamma \neq 1$

$$
\Delta_{\alpha}^{\prime}(\rho, \sigma, \mathcal{N}) \leq \Delta_{\gamma}^{\prime}(\rho, \sigma, \mathcal{N})
$$

Proof. The main tool for our proof is Theorem [3. We break the proof of inequality in (4.21) into several cases. We first consider $1<\alpha<\gamma \leq 2$. For some $W_{\mathcal{N}(\sigma)} \in \mathbb{V}_{\mathcal{N}(\sigma)}$ and $W_{\sigma} \in \mathbb{V}_{\sigma}$, pick

$$
\begin{aligned}
G(z) & =[\mathcal{N}(\rho)]^{-z(\gamma-1) / 2} W_{\mathcal{N}(\sigma)}[\mathcal{N}(\sigma)]^{z(\gamma-1) / 2} U \sigma^{-z(\gamma-1) / 2} W_{\sigma} \rho^{(1+z(\gamma-1)) / 2} \\
p_{0} & =2 \\
p_{1} & =2 \\
\theta & =\frac{\alpha-1}{\gamma-1} \in(0,1)
\end{aligned}
$$


which fixes $p_{\theta}=2$. Then

$$
\begin{aligned}
M_{0} & =\sup _{t \in \mathbb{R}}\|G(i t)\|_{2} \\
& =\sup _{t \in \mathbb{R}}\left\|[\mathcal{N}(\rho)]^{-i t(\gamma-1) / 2} W_{\mathcal{N}(\sigma)}[\mathcal{N}(\sigma)]^{i t(\gamma-1) / 2} U \sigma^{-i t(\gamma-1) / 2} W_{\sigma} \rho^{(1+i t(\gamma-1)) / 2}\right\|_{2} \\
& =\left\|\rho^{1 / 2}\right\|_{2}=1 \\
M_{1} & =\sup _{t \in \mathbb{R}}\|G(1+i t)\|_{2} \\
& =\sup _{t \in \mathbb{R}}\left\|[\mathcal{N}(\rho)]^{-\frac{(1+i t)}{2}(\gamma-1)} W_{\mathcal{N}(\sigma)}[\mathcal{N}(\sigma)]^{\frac{(1+i t)}{2}(\gamma-1)} U \sigma^{-\frac{(1+i t)}{2}(\gamma-1)} W_{\sigma} \rho^{\frac{(1+(1+i t)(\gamma-1))}{2}}\right\|_{2} \\
& \leq \max _{V_{\mathcal{N}(\sigma)}, V_{\sigma}}\left\|[\mathcal{N}(\rho)]^{(1-\gamma) / 2} V_{\mathcal{N}(\sigma)}[\mathcal{N}(\sigma)]^{(\gamma-1) / 2} U \sigma^{(1-\gamma) / 2} V_{\sigma} \rho^{\gamma / 2}\right\|_{2} \\
& =\exp \left\{\frac{\gamma-1}{2} \Delta_{\gamma}^{\prime}(\rho, \sigma, \mathcal{N})\right\} \\
\|G(\theta)\|_{2} & =\left\|[\mathcal{N}(\rho)]^{(1-\alpha) / 2} W_{\mathcal{N}(\sigma)}[\mathcal{N}(\sigma)]^{(\alpha-1) / 2} U \sigma^{(1-\alpha) / 2} W_{\sigma} \rho^{\alpha / 2}\right\|_{2}
\end{aligned}
$$

We then apply Theorem 3 to find that the following inequality holds for all $W_{\mathcal{N}(\sigma)} \in \mathbb{V}_{\mathcal{N}(\sigma)}$ and $W_{\sigma} \in \mathbb{V}_{\sigma}$ :

$$
\left\|[\mathcal{N}(\rho)]^{(1-\alpha) / 2} W_{\mathcal{N}(\sigma)}[\mathcal{N}(\sigma)]^{(\alpha-1) / 2} U \sigma^{(1-\alpha) / 2} W_{\sigma} \rho^{\alpha / 2}\right\|_{2} \leq\left[\exp \left\{\frac{\gamma-1}{2} \Delta_{\gamma}^{\prime}(\rho, \sigma, \mathcal{N})\right\}\right]^{\frac{\alpha-1}{\gamma-1}} .
$$

As a consequence, we can take the maximum over all $W_{\mathcal{N}(\sigma)} \in \mathbb{V}_{\mathcal{N}(\sigma)}$ and $W_{\sigma} \in \mathbb{V}_{\sigma}$ and apply the definition in (4.1) to establish that

$$
\exp \left\{\frac{\alpha-1}{2} \Delta_{\alpha}^{\prime}(\rho, \sigma, \mathcal{N})\right\} \leq\left[\exp \left\{\frac{\gamma-1}{2} \Delta_{\gamma}^{\prime}(\rho, \sigma, \mathcal{N})\right\}\right]^{\frac{\alpha-1}{\gamma-1}} .
$$

We finally apply a logarithm to arrive at the conclusion that (4.21) holds for all $1<\alpha<\gamma \leq 2$.

To get the monotonicity for the range $0 \leq \alpha<\gamma<1$, we exchange $\alpha$ and $\gamma$ in (4.22)-(4.25) and apply the same reasoning as in (4.26)-(4.34) to arrive at the following inequality:

$$
\exp \left\{\frac{\gamma-1}{2} \Delta_{\gamma}^{\prime}(\rho, \sigma, \mathcal{N})\right\} \leq\left[\exp \left\{\frac{\alpha-1}{2} \Delta_{\alpha}^{\prime}(\rho, \sigma, \mathcal{N})\right\}\right]^{\frac{\gamma-1}{\alpha-1}} .
$$

Taking a negative logarithm and noting that $0 \leq \alpha<\gamma<1$ then gives (4.21) for this range.

We are now left with proving the case $\alpha \in[0,1)$ and $\gamma \in(1,2]$ the dual parameter of $\alpha$, such that $\alpha+\gamma=2$. Notice that $\alpha-1=-(\gamma-1)$. Let $f(z, \gamma)=(1-2 z)(\gamma-1)$. We pick

$$
\begin{aligned}
G(z) & =[\mathcal{N}(\rho)]^{-f(z, \gamma) / 2}[\mathcal{N}(\sigma)]^{f(z, \gamma) / 2} U \sigma^{-f(z, \gamma) / 2} \rho^{(1+f(z, \gamma)) / 2}, \\
p_{0} & =2, \\
p_{1} & =2, \\
\theta & =1 / 2,
\end{aligned}
$$


so that $p_{\theta}=2$. Consider that $f(\theta, \gamma)=0$, so that

$$
\begin{aligned}
\|G(\theta)\|_{2} & =\left\|[\mathcal{N}(\rho)]^{-f(\theta, \gamma) / 2}[\mathcal{N}(\sigma)]^{f(\theta, \gamma) / 2} U \sigma^{-f(\theta, \gamma) / 2} \rho^{(1+f(\theta, \gamma)) / 2}\right\|_{2} \\
& =\left\|U \rho^{1 / 2}\right\|_{2}=\left\|\rho^{1 / 2}\right\|_{2}=1
\end{aligned}
$$

We then find that

$$
\begin{aligned}
M_{0} & =\sup _{t \in \mathbb{R}}\|G(i t)\|_{2} \\
& =\sup _{t \in \mathbb{R}}\left\|[\mathcal{N}(\rho)]^{-(1-2 i t)(\gamma-1) / 2}[\mathcal{N}(\sigma)]^{(1-2 i t)(\gamma-1) / 2} U \sigma^{-(1-2 i t)(\gamma-1) / 2} \rho^{(1+(1-2 i t)(\gamma-1)) / 2}\right\|_{2} \\
& \leq \max _{V_{\mathcal{N}(\sigma),}, V_{\sigma}}\left\|[\mathcal{N}(\rho)]^{(1-\gamma) / 2} V_{\mathcal{N}(\sigma)}[\mathcal{N}(\sigma)]^{(\gamma-1) / 2} U \sigma^{(1-\gamma) / 2} V_{\sigma} \rho^{\gamma / 2}\right\|_{2} \\
& =\exp \left\{\frac{\gamma-1}{2} \Delta_{\gamma}^{\prime}(\rho, \sigma, \mathcal{N})\right\} .
\end{aligned}
$$

Consider that

$$
f(1+i t, \gamma)=(1-2(1+i t))(\gamma-1)=-(1+2 i t)(\gamma-1)=(1+2 i t)(\alpha-1)
$$

Thus, similarly, we have

$$
\begin{aligned}
& M_{1}=\sup _{t \in \mathbb{R}}\|G(1+i t)\|_{2} \\
& =\sup _{t \in \mathbb{R}}\left\|[\mathcal{N}(\rho)]^{-(1+2 i t)(\alpha-1) / 2}[\mathcal{N}(\sigma)]^{(1+2 i t)(\alpha-1) / 2} U \sigma^{-(1+2 i t)(\alpha-1) / 2} \rho^{(1+(1+2 i t)(\alpha-1)) / 2}\right\|_{2} \\
& \leq \max _{V_{\mathcal{N}(\sigma)}, V_{\sigma}}\left\|[\mathcal{N}(\rho)]^{(1-\alpha) / 2} V_{\mathcal{N}(\sigma)}[\mathcal{N}(\sigma)]^{(\alpha-1) / 2} U \sigma^{(1-\alpha) / 2} V_{\sigma} \rho^{\alpha / 2}\right\|_{2} \\
& =\exp \left\{\frac{\alpha-1}{2} \Delta_{\alpha}^{\prime}(\rho, \sigma, \mathcal{N})\right\} \text {. }
\end{aligned}
$$

Applying Theorem 3 gives

$$
\begin{aligned}
1 & \leq \exp \left\{\frac{\gamma-1}{4} \Delta_{\gamma}^{\prime}(\rho, \sigma, \mathcal{N})\right\} \exp \left\{\frac{\alpha-1}{4} \Delta_{\alpha}^{\prime}(\rho, \sigma, \mathcal{N})\right\} \\
& =\exp \left\{\frac{\gamma-1}{4} \Delta_{\gamma}^{\prime}(\rho, \sigma, \mathcal{N})\right\} \exp \left\{\frac{-(\gamma-1)}{4} \Delta_{\alpha}^{\prime}(\rho, \sigma, \mathcal{N})\right\}
\end{aligned}
$$

which implies (4.21) for $\alpha \in[0,1)$ and $\gamma=2-\alpha$. Putting the three cases together along with Proposition 5 gives the inequality in (4.21) for $0 \leq \alpha \leq \gamma \leq 2, \alpha \neq 1$, and $\gamma \neq 1$.

Theorem 7 Let $\rho, \sigma$, and $\mathcal{N}$ be as given in Definition 1 . The swiveled Rényi quantity $\widetilde{\Delta}_{\alpha}^{\prime}(\rho, \sigma, \mathcal{N})$ is monotone non-decreasing with respect to $\alpha \in[1 / 2,1) \cup(1, \infty]$, in the sense that for $1 / 2 \leq \alpha \leq$ $\gamma \leq \infty, \alpha \neq 1$, and $\gamma \neq 1$

$$
\widetilde{\Delta}_{\alpha}^{\prime}(\rho, \sigma, \mathcal{N}) \leq \widetilde{\Delta}_{\gamma}^{\prime}(\rho, \sigma, \mathcal{N})
$$


Proof. We handle the inequality in (4.54) in a similar way as in the previous proof. First, suppose that $1<\alpha<\gamma$. Let $\alpha^{\prime}=(\alpha-1) / \alpha$ and $\gamma^{\prime}=(\gamma-1) / \gamma$, and note that $\alpha^{\prime}, \gamma^{\prime}>0$ for the choices given. For some $W_{\mathcal{N}(\sigma)} \in \mathbb{V}_{\mathcal{N}(\sigma)}$ and $W_{\sigma} \in \mathbb{V}_{\sigma}$, pick

$$
\begin{aligned}
G(z) & =[\mathcal{N}(\rho)]^{-z \gamma^{\prime} / 2} W_{\mathcal{N}(\sigma)}[\mathcal{N}(\sigma)]^{z \gamma^{\prime} / 2} U \sigma^{-z \gamma^{\prime} / 2} W_{\sigma} \rho^{1 / 2}, \\
p_{0} & =2 \\
p_{1} & =2 \gamma \\
\theta & =\frac{\alpha^{\prime}}{\gamma^{\prime}} \in(0,1)
\end{aligned}
$$

which fixes $p_{\theta}=2 \alpha$. Then we find the following expression for $M_{0}$

$$
\begin{aligned}
M_{0} & =\sup _{t \in \mathbb{R}}\|G(i t)\|_{2} \\
& =\sup _{t \in \mathbb{R}}\left\|[\mathcal{N}(\rho)]^{-i t \gamma^{\prime} / 2} W_{\mathcal{N}(\sigma)}[\mathcal{N}(\sigma)]^{i t \gamma^{\prime} / 2} U \sigma^{-i t \gamma^{\prime} / 2} W_{\sigma} \rho^{1 / 2}\right\|_{2} \\
& =\left\|\rho^{1 / 2}\right\|_{2}=1,
\end{aligned}
$$

and the following ones for $M_{1}$ and $\|G(\theta)\|_{2 \alpha}$ :

$$
\begin{aligned}
M_{1} & =\sup _{t \in \mathbb{R}}\|G(1+i t)\|_{2 \gamma} \\
& =\sup _{t \in \mathbb{R}}\left\|[\mathcal{N}(\rho)]^{-(1+i t) \gamma^{\prime} / 2} W_{\mathcal{N}(\sigma)}[\mathcal{N}(\sigma)]^{(1+i t) \gamma^{\prime} / 2} U \sigma^{-(1+i t) \gamma^{\prime} / 2} W_{\sigma} \rho^{1 / 2}\right\|_{2 \gamma} \\
& \leq \max _{V_{\mathcal{N}(\sigma)}, V_{\sigma}}\left\|[\mathcal{N}(\rho)]^{-\gamma^{\prime} / 2} V_{\mathcal{N}(\sigma)}[\mathcal{N}(\sigma)]^{\gamma^{\prime} / 2} U \sigma^{-\gamma^{\prime} / 2} V_{\sigma} \rho^{1 / 2}\right\|_{2 \gamma} \\
& =\exp \left\{\frac{\gamma^{\prime}}{2} \widetilde{\Delta}_{\gamma}^{\prime}(\rho, \sigma, \mathcal{N})\right\}, \\
\|G(\theta)\|_{2 \alpha} & =\left\|[\mathcal{N}(\rho)]^{-\alpha^{\prime} / 2} W_{\mathcal{N}(\sigma)}[\mathcal{N}(\sigma)]^{\alpha^{\prime} / 2} U \sigma^{-\alpha^{\prime} / 2} W_{\sigma} \rho^{1 / 2}\right\|_{2 \alpha} .
\end{aligned}
$$

Applying Theorem 3, we find that the following inequality holds for all $W_{\mathcal{N}(\sigma)} \in \mathbb{V}_{\mathcal{N}(\sigma)}$ and $W_{\sigma} \in$ $\mathbb{V}_{\sigma}$ :

$$
\left\|[\mathcal{N}(\rho)]^{-\alpha^{\prime} / 2} W_{\mathcal{N}(\sigma)}[\mathcal{N}(\sigma)]^{\alpha^{\prime} / 2} U \sigma^{-\alpha^{\prime} / 2} W_{\sigma} \rho^{1 / 2}\right\|_{2 \alpha} \leq\left[\exp \left\{\frac{\gamma^{\prime}}{2} \widetilde{\Delta}_{\gamma}^{\prime}(\rho, \sigma, \mathcal{N})\right\}\right]^{\frac{\alpha^{\prime}}{\gamma^{\prime}}}
$$

We can then take a maximum over all $W_{\mathcal{N}(\sigma)} \in \mathbb{V}_{\mathcal{N}(\sigma)}$ and $W_{\sigma} \in \mathbb{V}_{\sigma}$ and apply the definition in (4.2) to establish that

$$
\exp \left\{\frac{\alpha^{\prime}}{2} \widetilde{\Delta}_{\alpha}^{\prime}(\rho, \sigma, \mathcal{N})\right\} \leq\left[\exp \left\{\frac{\gamma^{\prime}}{2} \widetilde{\Delta}_{\gamma}^{\prime}(\rho, \sigma, \mathcal{N})\right\}\right]^{\frac{\alpha^{\prime}}{\gamma^{\prime}}} .
$$

The inequality in (4.54) then follows for $1<\alpha<\gamma$ after taking a logarithm.

To get the monotonicity for the range $1 / 2 \leq \alpha<\gamma<1$, we exchange $\alpha$ and $\gamma$ in (4.55)-(4.58) and apply the same reasoning as in (4.59)-(4.67) to arrive at the following inequality:

$$
\exp \left\{\frac{\gamma^{\prime}}{2} \widetilde{\Delta}_{\gamma}^{\prime}(\rho, \sigma, \mathcal{N})\right\} \leq\left[\exp \left\{\frac{\alpha^{\prime}}{2} \widetilde{\Delta}_{\alpha}^{\prime}(\rho, \sigma, \mathcal{N})\right\}\right]^{\frac{\gamma^{\prime}}{\alpha^{\prime}}}
$$


Taking a negative logarithm and noting that $1 / 2 \leq \alpha<\gamma<1$, so that $\alpha^{\prime}, \gamma^{\prime} \in[-1,0)$, then gives (4.54) for this range.

We are now left with proving the case $\alpha \in[1 / 2,1)$ and $\gamma \in(1, \infty]$ the dual parameter of $\alpha$ : such that $1 / \alpha+1 / \gamma=2$. Notice that $\alpha^{\prime}=-\gamma^{\prime}$ and we have that $\gamma^{\prime}>0$. We pick

$$
\begin{aligned}
G(z) & =[\mathcal{N}(\rho)]^{-(1-2 z) \alpha^{\prime} / 2}[\mathcal{N}(\sigma)]^{(1-2 z) \alpha^{\prime} / 2} U \sigma^{-(1-2 z) \alpha^{\prime} / 2} \rho^{1 / 2}, \\
p_{0} & =2 \alpha \\
p_{1} & =2 \gamma \\
\theta & =1 / 2,
\end{aligned}
$$

so that $p_{\theta}=2$. Consider that

$$
\begin{aligned}
\|G(\theta)\|_{2} & =\left\|[\mathcal{N}(\rho)]^{-(1-2 \theta) \alpha^{\prime} / 2}[\mathcal{N}(\sigma)]^{(1-2 \theta) \alpha^{\prime} / 2} U \sigma^{-(1-2 \theta) \alpha^{\prime} / 2} \rho^{1 / 2}\right\|_{2} \\
& =\left\|U \rho^{1 / 2}\right\|_{2}=\left\|\rho^{1 / 2}\right\|_{2}=1 .
\end{aligned}
$$

We then find that

$$
\begin{aligned}
M_{0} & =\sup _{t \in \mathbb{R}}\|G(i t)\|_{2 \alpha} \\
& =\sup _{t \in \mathbb{R}}\left\|[\mathcal{N}(\rho)]^{-(1-2 i t) \alpha^{\prime} / 2}[\mathcal{N}(\sigma)]^{(1-2 i t) \alpha^{\prime} / 2} U \sigma^{-(1-2 i t) \alpha^{\prime} / 2} \rho^{1 / 2}\right\|_{2 \alpha} \\
& \leq \max _{V_{\mathcal{N}(\sigma),}, V_{\sigma}}\left\|[\mathcal{N}(\rho)]^{-\alpha^{\prime} / 2} V_{\mathcal{N}(\sigma)}[\mathcal{N}(\sigma)]^{\alpha^{\prime} / 2} U \sigma^{-\alpha^{\prime} / 2} V_{\sigma} \rho^{1 / 2}\right\|_{2 \alpha} \\
& =\exp \left\{\frac{\alpha^{\prime}}{2} \widetilde{\Delta}_{\alpha}^{\prime}(\rho, \sigma, \mathcal{N})\right\} .
\end{aligned}
$$

Consider that

$$
(1-2(1+i t)) \alpha^{\prime}=-(1+2 i t) \alpha^{\prime}=(1+2 i t) \gamma^{\prime}
$$

Thus, similarly, we have

$$
\begin{aligned}
M_{1} & =\sup _{t \in \mathbb{R}}\|G(1+i t)\|_{2 \gamma} \\
& =\sup _{t \in \mathbb{R}}\left\|[\mathcal{N}(\rho)]^{-(1+2 i t) \gamma^{\prime} / 2}[\mathcal{N}(\sigma)]^{(1+2 i t) \gamma^{\prime} / 2} U \sigma^{-(1+2 i t) \gamma^{\prime} / 2} \rho^{1 / 2}\right\|_{2 \gamma} \\
& \leq \max _{V_{\mathcal{N}(\sigma),}, V_{\sigma}}\left\|[\mathcal{N}(\rho)]^{-\gamma^{\prime} / 2} V_{\mathcal{N}(\sigma)}[\mathcal{N}(\sigma)]^{\gamma^{\prime} / 2} U \sigma^{-\gamma^{\prime} / 2} V_{\sigma} \rho^{1 / 2}\right\|_{2 \gamma} \\
& =\exp \left\{\frac{\gamma^{\prime}}{2} \widetilde{\Delta}_{\gamma}^{\prime}(\rho, \sigma, \mathcal{N})\right\} .
\end{aligned}
$$

Applying Theorem 3 gives

$$
\begin{aligned}
1 & \leq \exp \left\{\frac{\alpha^{\prime}}{4} \widetilde{\Delta}_{\alpha}^{\prime}(\rho, \sigma, \mathcal{N})\right\} \exp \left\{\frac{\gamma^{\prime}}{4} \widetilde{\Delta}_{\gamma}^{\prime}(\rho, \sigma, \mathcal{N})\right\} \\
& =\exp \left\{-\frac{\gamma^{\prime}}{4} \widetilde{\Delta}_{\alpha}^{\prime}(\rho, \sigma, \mathcal{N})\right\} \exp \left\{\frac{\gamma^{\prime}}{4} \widetilde{\Delta}_{\gamma}^{\prime}(\rho, \sigma, \mathcal{N})\right\},
\end{aligned}
$$

which implies (4.54) for $\alpha \in[1 / 2,1$ ) and $1 / \gamma=2-1 / \alpha$. Putting the three cases together along with Proposition 5 gives the inequality in (4.54) for $1 / 2 \leq \alpha \leq \gamma \leq \infty, \alpha \neq 1$, and $\gamma \neq 1$. 


\subsection{Bounds for the quantum relative entropy difference}

A recent work Wil15 established refinements of the monotonicity of quantum relative entropy, strong subadditivity, and other entropy inequalities. In this section, we point out that these results follow as a consequence of the properties of the swiveled Rényi entropies and along the way establish two new refinements of these entropy inequalities.

We begin with a brief background. Let $\mathcal{P}_{\sigma, \mathcal{N}}$ denote the Petz recovery map [Pet86b, Pet88] (see also [BK02]):

$$
\mathcal{P}_{\sigma, \mathcal{N}}(\cdot) \equiv \sigma^{1 / 2} \mathcal{N}^{\dagger}\left([\mathcal{N}(\sigma)]^{-1 / 2}(\cdot)[\mathcal{N}(\sigma)]^{-1 / 2}\right) \sigma^{1 / 2}
$$

and let $\mathcal{R}_{\sigma, \mathcal{N}}^{V, W}$ denote the swiveled Petz recovery map

$$
\mathcal{R}_{\sigma, \mathcal{N}}^{V, W}(\cdot) \equiv\left(\mathcal{W}_{\sigma} \circ \mathcal{P}_{\sigma, \mathcal{N}} \circ \mathcal{V}_{\mathcal{N}(\sigma)}\right)(\cdot)
$$

where the partial isometric map $\mathcal{V}_{\mathcal{N}(\sigma)}$ is defined by

$$
\mathcal{V}_{\mathcal{N}(\sigma)}(\cdot)=V_{\mathcal{N}(\sigma)}(\cdot) V_{\mathcal{N}(\sigma)}^{\dagger},
$$

and similarly for $\mathcal{W}_{\sigma}$, so that $\mathcal{V}_{\mathcal{N}(\sigma)}(\mathcal{N}(\sigma))=\mathcal{N}(\sigma)$ and $\mathcal{W}_{\sigma}(\sigma)=\sigma$. Observe then that

$$
\mathcal{R}_{\sigma, \mathcal{N}}^{V, W}(\mathcal{N}(\sigma))=\sigma .
$$

Consider that particular values of $\alpha$ for $\Delta_{\alpha}^{\prime}(\rho, \sigma, \mathcal{N})$ and $\widetilde{\Delta}_{\alpha}^{\prime}(\rho, \sigma, \mathcal{N})$ lead to the following quantities, which can be interpreted as a (pseudo-)distance from the state $\rho$ to the state $\mathcal{N}(\rho)$ after a recovery channel $\mathcal{R}_{\sigma, \mathcal{N}}^{V, W}$ is applied:

$$
\begin{aligned}
\Delta_{0}^{\prime}(\rho, \sigma, \mathcal{N}) & =\min _{V_{\mathcal{N}(\sigma), W_{\sigma}}} D_{0}\left(\rho \| \mathcal{R}_{\sigma, \mathcal{N}}^{V, W}(\mathcal{N}(\rho))\right), \\
\widetilde{\Delta}_{1 / 2}^{\prime}(\rho, \sigma, \mathcal{N}) & =-\log \max _{V_{\mathcal{N}(\sigma)}, W_{\sigma}} F\left(\rho, \mathcal{R}_{\sigma, \mathcal{N}}^{V, W}(\mathcal{N}(\rho))\right) .
\end{aligned}
$$

These observations combined with the monotonicity from Theorems 6 and 7 and the facts that $D_{0}\left(\rho \| \mathcal{R}_{\sigma, \mathcal{N}}^{V, W}(\mathcal{N}(\rho))\right) \geq 0$ and $-\log \max _{V_{\mathcal{N}(\sigma)}, W_{\sigma}} F\left(\rho, \mathcal{R}_{\sigma, \mathcal{N}}^{V, W}(\mathcal{N}(\rho))\right) \geq 0$ allow us to conclude the following:

Corollary 8 Let $\rho, \sigma$, and $\mathcal{N}$ be as given in Definition 1 . The swiveled Rényi quantity $\Delta_{\alpha}^{\prime}(\rho, \sigma, \mathcal{N})$ is non-negative for $\alpha \in[0,1) \cup(1,2]$ and $\widetilde{\Delta}_{\alpha}^{\prime}(\rho, \sigma, \mathcal{N})$ is non-negative for $\alpha \in[1 / 2,1) \cup(1, \infty]$.

In order to establish the upper bounds in this section, we need to take $\rho, \sigma$, and $\mathcal{N}$ as given in the following definition:

Definition 9 Let $\rho_{S E^{\prime}}$ be a positive definite density operator and let $\sigma_{S E^{\prime}}$ be a positive definite operator, each acting on a finite-dimensional tensor-product Hilbert space $\mathcal{H}_{S} \otimes \mathcal{H}_{E^{\prime}}$. Let $\mathcal{N}$ be a quantum channel given as follows:

$$
\mathcal{N}\left(\theta_{S E^{\prime}}\right)=\operatorname{Tr}_{E}\left\{U_{S E^{\prime} \rightarrow B E} \theta_{S E^{\prime}} U_{S E^{\prime} \rightarrow B E}^{\dagger}\right\}
$$

where $U_{S E^{\prime} \rightarrow B E}$ is a unitary operator taking $\mathcal{H}_{S} \otimes \mathcal{H}_{E^{\prime}}$ to an isomorphic finite-dimensional tensorproduct Hilbert space $\mathcal{H}_{B} \otimes \mathcal{H}_{E}$, such that $\mathcal{N}(\rho)$ and $\mathcal{N}(\sigma)$ are each positive definite and act on $\mathcal{H}_{B}$. 
If $\rho, \sigma$, and $\mathcal{N}$ are taken as in Definition [9, then the following relations hold

$$
\begin{aligned}
\Delta_{2}^{\prime}(\rho, \sigma, \mathcal{N}) & =\max _{V_{\mathcal{N}(\sigma)}, W_{\sigma}} D_{2}\left(\rho \| \mathcal{R}_{\sigma, \mathcal{N}}^{V, W}(\mathcal{N}(\rho))\right), \\
\widetilde{\Delta}_{\infty}^{\prime}(\rho, \sigma, \mathcal{N}) & =\max _{V_{\mathcal{N}(\sigma), W_{\sigma}}} D_{\max }\left(\rho \| \mathcal{R}_{\sigma, \mathcal{N}}^{V, W}(\mathcal{N}(\rho))\right) .
\end{aligned}
$$

The main contribution of the recent work Wil15] was to show that the relative entropy difference $\Delta(\rho, \sigma, \mathcal{N})$ in (3.3) can be bounded from below by (4.92). In the case that $\rho, \sigma$, and $\mathcal{N}$ are taken as in Definition 9, then $\Delta(\rho, \sigma, \mathcal{N})$ can be bounded from above by (4.95). We find here that these results are an immediate corollary of Proposition 5 and Theorem [6, and we also obtain two new bounds on $\Delta(\rho, \sigma, \mathcal{N})$ in terms of $\Delta_{0}(\rho, \sigma, \mathcal{N})$ and $\Delta_{2}(\rho, \sigma, \mathcal{N})$ :

Corollary 10 Let $\rho, \sigma$, and $\mathcal{N}$ be as given in Definition 1 and such that $\operatorname{supp}(\rho) \subseteq \operatorname{supp}(\sigma)$. Then the following inequalities hold

$$
\begin{aligned}
& -\log \max _{V_{\mathcal{N}(\sigma)}, W_{\sigma}} F\left(\rho, \mathcal{R}_{\sigma, \mathcal{N}}^{V, W}(\mathcal{N}(\rho))\right) \leq D(\rho \| \sigma)-D(\mathcal{N}(\rho) \| \mathcal{N}(\sigma)), \\
& \min _{V_{\mathcal{N}(\sigma)}, W_{\sigma}} D_{0}\left(\rho \| \mathcal{R}_{\sigma, \mathcal{N}}^{V, W}(\mathcal{N}(\rho))\right) \leq D(\rho \| \sigma)-D(\mathcal{N}(\rho) \| \mathcal{N}(\sigma)) .
\end{aligned}
$$

If $\rho, \sigma$, and $\mathcal{N}$ are as given in Definition 9 , then the following inequalities hold

$$
\begin{aligned}
& D(\rho \| \sigma)-D(\mathcal{N}(\rho) \| \mathcal{N}(\sigma)) \leq \max _{V_{\mathcal{N}(\sigma)}, W_{\sigma}} D_{\max }\left(\rho \| \mathcal{R}_{\sigma, \mathcal{N}}^{V, W}(\mathcal{N}(\rho))\right), \\
& D(\rho \| \sigma)-D(\mathcal{N}(\rho) \| \mathcal{N}(\sigma)) \leq \max _{V_{\mathcal{N}(\sigma)}, W_{\sigma}} D_{2}\left(\rho \| \mathcal{R}_{\sigma, \mathcal{N}}^{V, W}(\mathcal{N}(\rho))\right) .
\end{aligned}
$$

As discussed in Wil15] (see also [BLW15, SBW15]), Corollary 10 can be viewed as providing a physically meaningful refinement of the monotonicity of quantum relative entropy in (3.2). The bound

$$
-\log \max _{V_{\mathcal{N}(\sigma)}, W_{\sigma}} F\left(\rho, \mathcal{R}_{\sigma, \mathcal{N}}^{V, W}(\mathcal{N}(\rho))\right) \leq D(\rho \| \sigma)-D(\mathcal{N}(\rho) \| \mathcal{N}(\sigma))
$$

shows that if the decrease in relative entropy is small after the channel $\mathcal{N}$ acts, then it is possible to perform the recovery map $\mathcal{R}_{\sigma, \mathcal{N}}^{V, W}$ such that $\sigma$ is recovered perfectly from $\mathcal{N}(\sigma)$, while the recovery of $\rho$ from $\mathcal{N}(\rho)$ has a performance limited by the bound above. This result has far reaching implications in quantum information theory as discussed in [Wil15] (see also [BLW15, [SBW15]).

We mention here that it is also possible to obtain bounds of the form from [Wil15], with a single "time" variable $t \in \mathbb{R}$. The method of proof is similar to that for Theorem 4 in [Wil15], so we give it in Appendix B, The formal statement is as follows:

Theorem 11 Let $\rho, \sigma$, and $\mathcal{N}$ be as given in Definition 1 and such that $\operatorname{supp}(\rho) \subseteq \operatorname{supp}(\sigma)$. Then the following inequalities hold

$$
\inf _{t \in \mathbb{R}} D_{0}\left(\rho \| \mathcal{R}_{\sigma, \mathcal{N}}^{t}(\mathcal{N}(\rho))\right) \leq D(\rho \| \sigma)-D(\mathcal{N}(\rho) \| \mathcal{N}(\sigma)),
$$

where $\mathcal{R}_{\sigma, \mathcal{N}}^{t}$ is the following rotated Petz recovery map:

$$
\mathcal{R}_{\sigma, \mathcal{N}}^{t}(\cdot) \equiv\left(\mathcal{U}_{\sigma, t} \circ \mathcal{P}_{\sigma, \mathcal{N}} \circ \mathcal{U}_{\mathcal{N}(\sigma),-t}\right)(\cdot)
$$


$\mathcal{P}_{\sigma, \mathcal{N}}$ is the Petz recovery map defined in (4.87), and $\mathcal{U}_{\sigma, t}$ and $\mathcal{U}_{\mathcal{N}(\sigma),-t}$ are partial isometric maps defined from

$$
\mathcal{U}_{\omega, t}(\cdot) \equiv \omega^{i t}(\cdot) \omega^{-i t},
$$

with $\omega$ a positive semi-definite operator. If $\rho, \sigma$, and $\mathcal{N}$ are as given in Definition 9, then

$$
D(\rho \| \sigma)-D(\mathcal{N}(\rho) \| \mathcal{N}(\sigma)) \leq \sup _{t \in \mathbb{R}} D_{2}\left(\rho \| \mathcal{R}_{\sigma, \mathcal{N}}^{t}(\mathcal{N}(\rho))\right) .
$$

Remark 12 Note that it is possible to establish "universal" versions of the above inequalities, by employing Hirschman's improvement [Hir52] of the Hadamard three-line theorem, as done in $\left[J R S^{+} 15\right]$.

\section{Swiveled Rényi conditional mutual information}

In this section, we show how swiveled Rényi conditional mutual informations are special cases of the quantities defined in the previous section. Furthermore, they satisfy some of the properties that one would expect to hold for a Rényi generalization of the conditional mutual information. However, they generally do not converge to the conditional mutual information in the limit as $\alpha \rightarrow 1$.

Let $\rho_{A B C}$ be a density operator. Following from the observation [LW14] that

$$
I(A ; B \mid C)_{\rho}=\Delta(\rho, \sigma, \mathcal{N}),
$$

for the choices

$$
\rho=\rho_{A B C}, \quad \sigma=\rho_{A C} \otimes I_{B}, \quad \mathcal{N}=\operatorname{Tr}_{A},
$$

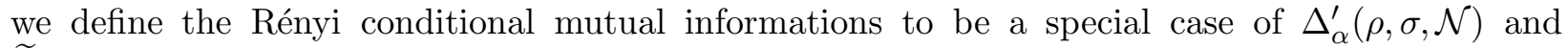
$\widetilde{\Delta}_{\alpha}^{\prime}(\rho, \sigma, \mathcal{N})$. That is, by setting

$$
\begin{aligned}
& I_{\alpha}^{\prime}(A ; B \mid C)_{\rho}=\Delta_{\alpha}^{\prime}\left(\rho_{A B C}, \rho_{A C} \otimes I_{B}, \operatorname{Tr}_{A}\right), \\
& \widetilde{I}_{\alpha}^{\prime}(A ; B \mid C)_{\rho}=\widetilde{\Delta}_{\alpha}^{\prime}\left(\rho_{A B C}, \rho_{A C} \otimes I_{B}, \operatorname{Tr}_{A}\right),
\end{aligned}
$$

we obtain the swiveled Rényi conditional mutual informations stated in the following definition:

Definition 13 The swiveled Rényi conditional mutual informations are defined for a density operator $\rho_{A B C}$ and $\alpha \in(0,1) \cup(1, \infty)$ as follows:

$$
\begin{aligned}
& I_{\alpha}^{\prime}(A ; B \mid C)_{\rho} \equiv \frac{2}{\alpha-1} \max _{V_{\rho_{A C}}, V_{\rho_{C}}} \log \left\|\rho_{B C}^{(1-\alpha) / 2} V_{\rho_{C}} \rho_{C}^{(\alpha-1) / 2} \rho_{A C}^{(1-\alpha) / 2} V_{\rho_{A C}} \rho_{A B C}^{\alpha / 2}\right\|_{2}, \\
& \widetilde{I}_{\alpha}^{\prime}(A ; B \mid C)_{\rho} \equiv \frac{2}{\alpha^{\prime}} \max _{V_{\rho_{A C}}, V_{\rho_{C}}} \log \left\|\rho_{B C}^{-\alpha^{\prime} / 2} V_{\rho_{C}} \rho_{C}^{\alpha^{\prime} / 2} \rho_{A C}^{-\alpha^{\prime} / 2} V_{\rho_{A C}} \rho_{A B C}^{1 / 2}\right\|_{2 \alpha},
\end{aligned}
$$

where $\alpha^{\prime}=(\alpha-1) / \alpha$.

We can now easily show that the Rényi conditional mutual informations as defined above satisfy several natural properties, with the exception of convergence to the von Neumann conditional mutual information.

The following is a consequence of (5.3)-(5.4) and Proposition 5: 
Corollary 14 Let $\rho_{A B C}$ be a positive definite density operator. Then

$\lim _{\alpha \nearrow 1} I_{\alpha}^{\prime}(A ; B \mid C)_{\rho}=\lim _{\alpha \nearrow 1} \widetilde{I}_{\alpha}^{\prime}(A ; B \mid C)_{\rho} \leq I(A ; B \mid C)_{\rho} \leq \lim _{\alpha \searrow 1} I_{\alpha}^{\prime}(A ; B \mid C)_{\rho}=\lim _{\alpha \searrow 1} \widetilde{I}_{\alpha}^{\prime}(A ; B \mid C)_{\rho}$.

They are monotone non-decreasing with respect to the parameter $\alpha$, which follows from (5.3)(5.4) and Theorems 6 and 7 .

Corollary 15 Let $\rho_{A B C}$ be a density operator. The swiveled Rényi conditional mutual informations $I_{\alpha}^{\prime}(A ; B \mid C)_{\rho}$ and $\widetilde{I}_{\alpha}^{\prime}(A ; B \mid C)_{\rho}$ are monotone non-decreasing with respect to the Rényi parameter for particular values. For $0 \leq \alpha \leq \gamma \leq 2, \alpha \neq 1$, and $\gamma \neq 1$, we have that

$$
I_{\alpha}^{\prime}(A ; B \mid C)_{\rho} \leq I_{\gamma}^{\prime}(A ; B \mid C)_{\rho}
$$

and for $1 / 2 \leq \alpha \leq \gamma \leq \infty, \alpha \neq 1$, and $\gamma \neq 1$,

$$
\widetilde{I}_{\alpha}^{\prime}(A ; B \mid C)_{\rho} \leq \widetilde{I}_{\gamma}^{\prime}(A ; B \mid C)_{\rho} .
$$

They are monotone non-increasing with respect to a quantum channel acting on the $B$ system, which follows by invoking [BSW15a, Lemmas 13 and 23]:

Corollary 16 Let $\rho_{A B C}$ be a positive definite density operator, and let $\mathcal{N}_{B \rightarrow B^{\prime}}$ be a quantum channel such that

$$
\sigma_{A B^{\prime} C} \equiv \mathcal{N}_{B \rightarrow B^{\prime}}\left(\rho_{A B C}\right)
$$

is a positive definite density operator. Then for all $\alpha \in[0,1) \cup(1,2]$, the following inequality holds

$$
I_{\alpha}^{\prime}(A ; B \mid C)_{\rho} \geq I_{\alpha}^{\prime}\left(A ; B^{\prime} \mid C\right)_{\sigma},
$$

and for all $\alpha \in[1 / 2,1) \cup(1, \infty]$, the following inequality holds

$$
\widetilde{I}_{\alpha}^{\prime}(A ; B \mid C)_{\rho} \geq \widetilde{I}_{\alpha}^{\prime}\left(A ; B^{\prime} \mid C\right)_{\sigma} .
$$

Corollary 15, Proposition 5, and (5.3) -(5.4) then imply the following refinements of the strong subaddivity of quantum entropy, two of which were already determined in [Wil15:

Corollary 17 Let $\rho_{A B C}$ be a density operator. Then the following inequalities hold

$$
\begin{aligned}
-\log \left[\max _{W_{\rho_{C}}, V_{\rho_{A C}}} F\left(\rho_{A B C}, \mathcal{R}_{C \rightarrow A C}^{V, W}\left(\rho_{B C}\right)\right)\right] & \leq I(A ; B \mid C)_{\rho}, \\
\min _{W_{\rho_{C}}, V_{\rho_{A C}}} D_{0}\left(\rho_{A B C} \| \mathcal{R}_{C \rightarrow A C}^{V, W}\left(\rho_{B C}\right)\right) & \leq I(A ; B \mid C)_{\rho},
\end{aligned}
$$

where $\mathcal{R}_{C \rightarrow A C}^{V, W}$ is the following swiveled Petz recovery map:

$$
\mathcal{R}_{C \rightarrow A C}^{V, W}(\cdot) \equiv\left(\mathcal{V}_{\rho_{A C}} \circ \mathcal{P}_{C \rightarrow A C} \circ \mathcal{W}_{\rho_{C}}\right)(\cdot),
$$

the Petz recovery map $\mathcal{P}_{C \rightarrow A C}$ is defined as

$$
\mathcal{P}_{C \rightarrow A C}(\cdot) \equiv \mathcal{P}_{\rho_{A C}, \operatorname{Tr}_{A}}(\cdot)=\rho_{A C}^{1 / 2} \rho_{C}^{-1 / 2}(\cdot) \rho_{C}^{-1 / 2} \rho_{A C}^{1 / 2},
$$


and the partial isometric maps $\mathcal{V}_{\rho_{A C}}$ and $\mathcal{W}_{\rho_{C}}$ are defined as in (4.89). If $\rho_{A B C}$ is a positive definite density operator, then the following inequalities hold

$$
\begin{aligned}
& I(A ; B \mid C)_{\rho} \leq \max _{W_{\rho_{C}}, V_{\rho_{A C}}} D_{\max }\left(\rho_{A B C} \| \mathcal{R}_{C \rightarrow A C}^{V, W}\left(\rho_{B C}\right)\right), \\
& I(A ; B \mid C)_{\rho} \leq \max _{W_{\rho_{C}}, V_{\rho_{A C}}} D_{2}\left(\rho_{A B C} \| \mathcal{R}_{C \rightarrow A C}^{V, W}\left(\rho_{B C}\right)\right) .
\end{aligned}
$$

Note that remainder terms for strong subadditivity were put forward in [FR15, SFR15] before the recent developments in [Wil15.

\section{Swiveled Rényi quantum information measures}

We now discuss how to extend the approach given here and in BSW15b] in order to construct swiveled Rényi generalizations of any quantum information measure which consists of a linear combination of von Neumann entropies with coefficients chosen from the set $\{-1,0,1\}$. We repeat some of the discussions from [BSW15b] in order to illustrate the method.

Let $\rho_{A_{1} \cdots A_{l}}$ be a density operator on $l$ systems and set $\mathcal{A} \equiv\left\{A_{1}, \ldots, A_{l}\right\}$. Suppose that we would like to establish a Rényi generalization of the following linear combination of entropies:

$$
\left.L\left(\rho_{A_{1} \cdots A_{l}}\right) \equiv \sum_{S \in \mathcal{P} \geq 1} a_{S} H(S)\right)_{\rho}
$$

where $\mathcal{P}_{\geq 1}(\mathcal{A})$ is the power set of $\mathcal{A}$ (excluding the empty set), such that the sum runs over all subsets of the systems $A_{1}, \ldots, A_{l}$. Furthermore, each coefficient $a_{S} \in\{-1,0,1\}$ and corresponds to a subset $S$. In the case that $a_{\mathcal{A}}$ is nonzero, without loss of generality, we can set $a_{\mathcal{A}}=-1$ (otherwise, factor out -1 to make this the case). Then, we can rewrite the quantity in (6.1) in terms of the relative entropy as follows:

$$
D\left(\rho_{A_{1} \cdots A_{l}} \| \exp \left\{\sum_{S \in \mathcal{P}^{\prime}} a_{S} \log \rho_{S}\right\}\right),
$$

where $\mathcal{P}^{\prime}=\mathcal{P}_{\geq 1}(\mathcal{A}) \backslash\left\{A_{1}, \ldots, A_{l}\right\}$. On the other hand, if $a_{\mathcal{A}}=0$, i.e., if all the marginal entropies in the sum are on a number of systems that is strictly smaller than the number of systems over which the state $\rho$ is defined (as is the case with $H(A B)+H(B C)+H(A C)$, for example), we can take a purification of the original state and call this purification the state $\rho_{A_{1} \cdots A_{l}}$. This state is now a pure state on a number of systems strictly larger than the number of systems involved in all the marginal entropies. We then add the entropy $H\left(A_{1} \ldots A_{l}\right)_{\rho}=0$ to the sum of entropies and apply the above recipe (so we resolve the issue with this example by purifying to a system $R$, setting the sum formula to be $H(A B C R)+H(A B)+H(B C)+H(A C)$, and proceeding with the above recipe). In the case that the resulting density operator $\rho_{A_{1} \cdots A_{l}}$ is not positive definite, we can mix it with the maximally mixed state $\pi_{A_{1} \cdots A_{l}}$ as follows:

$$
(1-\varepsilon) \rho_{A_{1} \cdots A_{l}}+\varepsilon \pi_{A_{1} \cdots A_{l}},
$$

where $\varepsilon \in(0,1)$. The resulting density operator is then $\varepsilon$-distinguishable from the original one by any quantum measurement performed on the systems $A_{1} \cdots A_{l}$. 
We then define the following swiveled Rényi entropies, which generalize $L\left(\rho_{A_{1} \cdots A_{l}}\right)$ from (6.1):

$$
\begin{aligned}
& L_{\alpha}^{\prime}\left(\rho_{A_{1} \cdots A_{l}}\right) \equiv \frac{2}{\alpha-1} \max _{\left\{V_{\rho_{S}}\right\}_{S}} \log \left\|\left[\prod_{S \in \mathcal{P}^{\prime}} \rho_{S}^{-a_{S}(\alpha-1) / 2} V_{\rho_{S}}\right] \rho_{A_{1} \cdots A_{l}}^{\alpha / 2}\right\|_{2}, \\
& \widetilde{L}_{\alpha}^{\prime}\left(\rho_{A_{1} \cdots A_{l}}\right) \equiv \frac{2}{\alpha^{\prime}} \max _{\left\{V_{\rho_{S}}\right\}_{S}} \log \left\|\left[\prod_{S \in \mathcal{P}^{\prime}} \rho_{S}^{-a_{S} \alpha^{\prime} / 2} V_{\rho_{S}}\right] \rho_{A_{1} \cdots A_{l}}^{1 / 2}\right\|_{2 \alpha},
\end{aligned}
$$

where $\alpha^{\prime}=(\alpha-1) / \alpha$. The ordering of the marginal density operators in the product is in general arbitrary, but could be important for some applications (consider, e.g., that the choices in Definition 13 lead to the inequalities in Corollary 17, which have a physical interpretation in terms of recovery).

By the same methods as given throughout this paper, we can establish several properties of these quantities. It follows from the generalized Lie-Trotter product formula [Suz85] and the method given in the proof of Proposition 5 that

$$
\lim _{\alpha \nearrow 1} L_{\alpha}^{\prime}\left(\rho_{A_{1} \cdots A_{l}}\right)=\lim _{\alpha \nearrow 1} \widetilde{L}_{\alpha}^{\prime}\left(\rho_{A_{1} \cdots A_{l}}\right) \leq L\left(\rho_{A_{1} \cdots A_{l}}\right) \leq \lim _{\alpha \searrow 1} L_{\alpha}^{\prime}\left(\rho_{A_{1} \cdots A_{l}}\right)=\lim _{\alpha \searrow 1} \widetilde{L}_{\alpha}^{\prime}\left(\rho_{A_{1} \cdots A_{l}}\right) .
$$

From the same method as given in the proof of Theorems 6 and 7 , for $0 \leq \alpha \leq \gamma \leq 2, \alpha \neq 1$, and $\gamma \neq 1$, we can conclude that

$$
L_{\alpha}^{\prime}\left(\rho_{A_{1} \cdots A_{l}}\right) \leq L_{\gamma}^{\prime}\left(\rho_{A_{1} \cdots A_{l}}\right),
$$

and for $1 / 2 \leq \alpha \leq \gamma \leq \infty, \alpha \neq 1$, and $\gamma \neq 1$,

$$
\widetilde{L}_{\alpha}^{\prime}\left(\rho_{A_{1} \cdots A_{l}}\right) \leq \widetilde{L}_{\gamma}^{\prime}\left(\rho_{A_{1} \cdots A_{l}}\right) .
$$

The inequalities above then lead to the following bounds for $L\left(\rho_{A_{1} \cdots A_{l}}\right)$ :

$$
\begin{aligned}
L_{0}^{\prime}\left(\rho_{A_{1} \cdots A_{l}}\right) & \leq L\left(\rho_{A_{1} \cdots A_{l}}\right) \leq L_{2}^{\prime}\left(\rho_{A_{1} \cdots A_{l}}\right), \\
\widetilde{L}_{1 / 2}^{\prime}\left(\rho_{A_{1} \cdots A_{l}}\right) & \leq L\left(\rho_{A_{1} \cdots A_{l}}\right) \leq \widetilde{L}_{\infty}^{\prime}\left(\rho_{A_{1} \cdots A_{l}}\right),
\end{aligned}
$$

which in some cases might have physical interpretations in terms of swiveled Petz recovery channels (see [Wil15, Sections 5.6 and 5.7] for some examples).

\section{Monotonicity of Trace Quantities}

[Zha14] posed an open question regarding whether the following quantity

$$
\operatorname{Tr}\left\{\left[\rho_{A C}^{(1-\alpha) / 2} \rho_{C}^{(\alpha-1) / 2} \rho_{B C}^{1-\alpha} \rho_{C}^{(\alpha-1) / 2} \rho_{A C}^{(1-\alpha) / 2}\right]^{1 /(1-\alpha)}\right\}
$$

is monotone in $\alpha$ and never exceeds one. The recent work [DW15] addressed this open question, first by generalizing it and then proving that

$$
\operatorname{Tr}\left\{\left[\sigma^{(1-\alpha) / 2} \mathcal{N}^{\dagger}\left(\mathcal{N}(\sigma)^{(\alpha-1) / 2} \mathcal{N}(\rho)^{1-\alpha} \mathcal{N}(\sigma)^{(\alpha-1) / 2}\right) \sigma^{(1-\alpha) / 2}\right]^{1 /(1-\alpha)}\right\} \leq 1,
$$

for $\alpha \in(0,1)$ and $\rho, \sigma$, and $\mathcal{N}$ as given in Definition 1, The same work established that this bound holds for $\alpha \in(1,2)$ if $\rho, \sigma$, and $\mathcal{N}$ are as given in Definition 11. One recovers the quantity in (7.1) 
by picking $\rho=\rho_{A B C}, \sigma=\rho_{A C} \otimes I_{B}$, and $\mathcal{N}=\operatorname{Tr}_{A}$ in (7.2). It is not known whether the quantity in (7.2) is monotone with respect to $\alpha$.

Here, we address this latter question by again taking our approach of allowing for a unitary swivel. Consider that we can rewrite the left-hand side of (7.2) as follows for $\alpha \in(0,1)$ :

$$
\left\|\left[\mathcal{N}(\rho)^{(1-\alpha) / 2} \mathcal{N}(\sigma)^{(\alpha-1) / 2} \otimes I_{E}\right] U \sigma^{(1-\alpha) / 2}\right\|_{2 /(1-\alpha)}^{2 /(1-\alpha)}
$$

where $U$ is an isometric extension of the channel $\mathcal{N}$. So we instead consider the following quantity, which has an optimization over a unitary swivel:

$$
\max _{V_{\mathcal{N}(\sigma)}}\left\|\left[\mathcal{N}(\rho)^{(1-\alpha) / 2} V_{\mathcal{N}(\sigma)} \mathcal{N}(\sigma)^{(\alpha-1) / 2} \otimes I_{E}\right] U \sigma^{(1-\alpha) / 2}\right\|_{2 /(1-\alpha)}^{2 /(1-\alpha)} .
$$

To simplify the notation, consider that the above quantity for $\alpha \in[0,1)$ is the same as the following one for $p \in[2, \infty)$ :

$$
\max _{V_{\mathcal{N}(\sigma)}}\left\|\left[\mathcal{N}(\rho)^{1 / p} V_{\mathcal{N}(\sigma)} \mathcal{N}(\sigma)^{-1 / p} \otimes I_{E}\right] U \sigma^{1 / p}\right\|_{p}^{p} .
$$

We can now state our contribution to the open question:

Proposition 18 The quantity in (7.5) is monotone non-increasing on the interval $p \in[2, \infty)$ and has a maximum value of one at $p=2$ if $\operatorname{supp}(\rho) \subseteq \operatorname{supp}(\sigma)$.

Proof. This ends up being another application of the Hadamard three-line theorem, using techniques similar to what we have used previously. For $q \in[2, \infty), q<p$, and $V_{\mathcal{N}(\sigma)}$ a fixed unitary commuting with $\mathcal{N}(\sigma)$, pick

$$
\begin{aligned}
G(z) & =\left[\mathcal{N}(\rho)^{z / q} V_{\mathcal{N}(\sigma)} \mathcal{N}(\sigma)^{-z / q} \otimes I_{E}\right] U \sigma^{z / q} \\
p_{0} & =\infty \\
p_{1} & =q \\
\theta & =q / p
\end{aligned}
$$

which implies that $p_{\theta}=p$. Applying Theorem 3 gives

$$
\|G(\theta)\|_{p} \leq\left[\sup _{t \in \mathbb{R}}\|G(i t)\|_{\infty}\right]^{1-\theta}\left[\sup _{t \in \mathbb{R}}\|G(1+i t)\|_{q}\right]^{\theta} .
$$


So we evaluate these terms to find

$$
\begin{aligned}
\|G(\theta)\|_{p} & =\left\|\left[\mathcal{N}(\rho)^{\theta / q} V_{\mathcal{N}(\sigma)} \mathcal{N}(\sigma)^{-\theta / q} \otimes I_{E}\right] U \sigma^{\theta / q}\right\|_{p} \\
& =\left\|\left[\mathcal{N}(\rho)^{1 / p} V_{\mathcal{N}(\sigma)} \mathcal{N}(\sigma)^{-1 / p} \otimes I_{E}\right] U \sigma^{1 / p}\right\|_{p} \\
\sup _{t \in \mathbb{R}}\|G(i t)\|_{\infty} & =\sup _{t \in \mathbb{R}}\left\|\left[\mathcal{N}(\rho)^{i t / q} V_{\mathcal{N}(\sigma)} \mathcal{N}(\sigma)^{-i t / q} \otimes I_{E}\right] U \sigma^{i t / q}\right\|_{\infty} \\
& \leq 1, \\
\sup _{t \in \mathbb{R}}\|G(1+i t)\|_{q} & =\sup _{t \in \mathbb{R}}\left\|\left[\mathcal{N}(\rho)^{(1+i t) / q} V_{\mathcal{N}(\sigma)} \mathcal{N}(\sigma)^{-(1+i t) / q} \otimes I_{E}\right] U \sigma^{(1+i t) / q}\right\|_{q} \\
& =\sup _{t \in \mathbb{R}}\left\|\left[\mathcal{N}(\rho)^{1 / q} V_{\mathcal{N}(\sigma)} \mathcal{N}(\sigma)^{-i t / q} \mathcal{N}(\sigma)^{-1 / q} \otimes I_{E}\right] U \sigma^{1 / q}\right\|_{q} \\
& \leq \max _{W_{\mathcal{N}(\sigma)}}\left\|\left[\mathcal{N}(\rho)^{1 / q} W_{\mathcal{N}(\sigma)} \mathcal{N}(\sigma)^{-1 / q} \otimes I_{E}\right] U \sigma^{1 / q}\right\|_{q} .
\end{aligned}
$$

Putting everything together, we find that for $2 \leq q<p$, the following inequality holds

$$
\begin{aligned}
\max _{V_{\mathcal{N}(\sigma)}} \|\left[\mathcal{N}(\rho)^{1 / p} V_{\mathcal{N}(\sigma)} \mathcal{N}(\sigma)^{-1 / p} \otimes I_{E}\right] & U \sigma^{1 / p} \|_{p}^{p} \\
& \leq \max _{W_{\mathcal{N}(\sigma)}}\left\|\left[\mathcal{N}(\rho)^{1 / q} W_{\mathcal{N}(\sigma)} \mathcal{N}(\sigma)^{-1 / q} \otimes I_{E}\right] U \sigma^{1 / q}\right\|_{q}^{q},
\end{aligned}
$$

since the inequality from (7.10) holds for all $V_{\mathcal{N}(\sigma)}$. This establishes the first statement in the proposition.

For the second statement, consider evaluating (7.5) at $p=2$ for any choice of $V_{\mathcal{N}(\sigma)}$ :

$$
\begin{aligned}
& \left\|\left[\mathcal{N}(\rho)^{1 / 2} V_{\mathcal{N}(\sigma)} \mathcal{N}(\sigma)^{-1 / 2} \otimes I_{E}\right] U \sigma^{1 / 2}\right\|_{2}^{2} \\
& =\operatorname{Tr}\left\{\sigma^{1 / 2} U^{\dagger}\left[\mathcal{N}(\sigma)^{-1 / 2} V_{\mathcal{N}(\sigma)}^{\dagger} \mathcal{N}(\rho) V_{\mathcal{N}(\sigma)} \mathcal{N}(\sigma)^{-1 / 2} \otimes I_{E}\right] U \sigma^{1 / 2}\right\} \\
& =\operatorname{Tr}\left\{\sigma^{1 / 2} \mathcal{N}^{\dagger}\left[\mathcal{N}(\sigma)^{-1 / 2} V_{\mathcal{N}(\sigma)}^{\dagger} \mathcal{N}(\rho) V_{\mathcal{N}(\sigma)} \mathcal{N}(\sigma)^{-1 / 2}\right] \sigma^{1 / 2}\right\} \\
& =\operatorname{Tr}\left\{\mathcal{N}(\sigma) \mathcal{N}(\sigma)^{-1 / 2} V_{\mathcal{N}(\sigma)}^{\dagger} \mathcal{N}(\rho) V_{\mathcal{N}(\sigma)} \mathcal{N}(\sigma)^{-1 / 2}\right\} \\
& =\operatorname{Tr}\left\{\Pi_{\mathcal{N}(\sigma)} V_{\mathcal{N}(\sigma)}^{\dagger} \mathcal{N}(\rho) V_{\mathcal{N}(\sigma)}\right\} \\
& =\operatorname{Tr}\left\{\Pi_{\mathcal{N}(\sigma)} \mathcal{N}(\rho)\right\} \\
& =1
\end{aligned}
$$

Corollary 19 Let $\rho_{A B C}$ be a density operator. Then the following quantity is monotone nonincreasing for $\alpha \in[0,1)$ and takes a maximum value of one at $\alpha=0$ :

$$
\max _{V_{\rho_{C}}} \operatorname{Tr}\left\{\left(\rho_{A C}^{(1-\alpha) / 2} V_{\rho_{C}} \rho_{C}^{(\alpha-1) / 2} \rho_{B C}^{1-\alpha} \rho_{C}^{(\alpha-1) / 2} V_{\rho_{C}}^{\dagger} \rho_{A C}^{(1-\alpha) / 2}\right)^{1 /(1-\alpha)}\right\} .
$$

If $\rho_{A B C}$ is a positive definite, then the same quantity is monotone non-decreasing for $\alpha \in(1,2]$ and takes a maximum value of one at $\alpha=2$. 
Proof. The first statement follows by applying Proposition 18 for the choices $\rho=\rho_{A B C}, \sigma=$ $\rho_{A C} \otimes I_{B}$, and $\mathcal{N}=\operatorname{Tr}_{A}$. The second statement follows because

$$
\begin{aligned}
\left(\rho_{A C}^{(1-\alpha) / 2} V_{\rho_{C}} \rho_{C}^{(\alpha-1) / 2} \rho_{B C}^{1-\alpha} \rho_{C}^{(\alpha-1) / 2} V_{\rho_{C}}^{\dagger} \rho_{A C}^{(1-\alpha) / 2}\right)^{1 /(1-\alpha)} & \\
& =\left(\rho_{A C}^{(1-\beta) / 2} V_{\rho_{C}} \rho_{C}^{(\beta-1) / 2} \rho_{B C}^{1-\beta} \rho_{C}^{(\beta-1) / 2} V_{\rho_{C}}^{\dagger} \rho_{A C}^{(1-\beta) / 2}\right)^{1 /(1-\beta)}
\end{aligned}
$$

for $\beta=2-\alpha \in(0,1)$ and then we apply the first statement.

\section{Conclusion}

The main contribution of this paper is a general method, the "swiveled Rényi entropic" approach, for constructing $\alpha$-Rényi generalizations of a quantum information measure that are monotone nondecreasing in the parameter $\alpha$. The swiveled Rényi entropies are discontinuous at $\alpha=1$ and do not converge to von Neumann entropy-based quantities in the limit as $\alpha \rightarrow 1$. We suspect that the swiveled Rényi entropies might be helpful in understanding refinements of quantum informationprocessing tasks, but this remains unclear due to the lack of convergence at $\alpha=1$. At the very least, the technique recovers the recent refinements [Wil15] of fundamental entropy inequalities such as monotonicity of quantum relative entropy [Lin75, Uhl77] and strong subadditivity [LR73b, LR73a], in addition to providing new refinements for these entropy inequalities.

The most important open question going forward from here is to determine Rényi entropies which satisfy all of the desiderata that one would have for Rényi generalizations of quantum information measures. We find it curious that with the proposal in [BSW15b], one can prove convergence to a von Neumann entropy-based quantity in the limit as $\alpha \rightarrow 1$, but we are still unable to establish monotonicity in $\alpha$. However, with the swiveled Rényi entropies proposed here, the situation is reversed.

One might also consider developing chain rules for the swiveled Rényi entropies, along the lines established in Dup15, but it is unclear how useful this would be in practice, given that the quantities do not generally converge at $\alpha=1$.

Acknowledgements. We are grateful to Salman Beigi for insightful discussions about the topic of this paper. FD acknowledges the support of the Czech Science Foundation GA CR project P202/12/1142 and the support of the EU FP7 under grant agreement no 323970 (RAQUEL). MMW is grateful to Stephanie Wehner and her group for hospitality during a research visit to TU Delft (May 2015), to Renato Renner and his group for the same during a visit to ETH Zurich (June 2015), and acknowledges support from startup funds from the Department of Physics and Astronomy at LSU, the NSF under Award No. CCF-1350397, and the DARPA Quiness Program through US Army Research Office award W31P4Q-12-1-0019.

\section{A Limit as $\alpha \rightarrow 1$}

Definition 20 Let $\rho, \sigma$, and $\mathcal{N}$ be as given in Definition 1. For $\alpha \in(0,1) \cup(1, \infty)$, let

$$
\Delta_{\alpha}(\rho, \sigma, \mathcal{N})=\frac{1}{\alpha-1} \log Q_{\alpha}(\rho, \sigma, \mathcal{N})
$$


where

$$
Q_{\alpha}(\rho, \sigma, \mathcal{N}) \equiv\left\|\left(\mathcal{N}(\rho)^{(1-\alpha) / 2} \mathcal{N}(\sigma)^{(\alpha-1) / 2} \otimes I_{E}\right) U \sigma^{(1-\alpha) / 2} \rho^{\alpha / 2}\right\|_{2}^{2} .
$$

Theorem 21 Let $\rho, \sigma$, and $\mathcal{N}$ be as given in Definition 1 and such that $\operatorname{supp}(\rho) \subseteq \operatorname{supp}(\sigma)$. The following limit holds

$$
\lim _{\alpha \rightarrow 1} \Delta_{\alpha}(\rho, \sigma, \mathcal{N})=D(\rho \| \sigma)-D(\mathcal{N}(\rho) \| \mathcal{N}(\sigma))
$$

Proof. Let $\Pi_{\omega}$ denote the projection onto the support of $\omega$. From the condition $\operatorname{supp}(\rho) \subseteq \operatorname{supp}(\sigma)$, it follows that $\operatorname{supp}(\mathcal{N}(\rho)) \subseteq \operatorname{supp}(\mathcal{N}(\sigma))$ [Ren05, Appendix B.4]. We can then conclude that

$$
\Pi_{\sigma} \Pi_{\rho}=\Pi_{\rho}, \quad \Pi_{\mathcal{N}(\rho)} \Pi_{\mathcal{N}(\sigma)}=\Pi_{\mathcal{N}(\rho)} .
$$

We also know that $\operatorname{supp}\left(U \rho U^{\dagger}\right) \subseteq \operatorname{supp}\left(\mathcal{N}(\rho) \otimes I_{E}\right)$ Ren05, Appendix B.4], so that

$$
\left(\Pi_{\mathcal{N}(\rho)} \otimes I_{E}\right) \Pi_{U \rho U^{\dagger}}=\Pi_{U \rho U^{\dagger}}
$$

When $\alpha=1$, we find from the above facts that

$$
\begin{aligned}
Q_{1}(\rho, \sigma, \mathcal{N}) & =\left\|\left(\Pi_{\mathcal{N}(\rho)} \Pi_{\mathcal{N}(\sigma)} \otimes I_{E}\right) U \Pi_{\sigma} \rho^{1 / 2}\right\|_{2}^{2} \\
& =\left\|\left(\Pi_{\mathcal{N}(\rho)} \otimes I_{E}\right) U \Pi_{\rho} \rho^{1 / 2}\right\|_{2}^{2} \\
& =\left\|\left(\Pi_{\mathcal{N}(\rho)} \otimes I_{E}\right) \Pi_{U \rho U^{\dagger}} U \rho^{1 / 2}\right\|_{2}^{2} \\
& =\left\|\Pi_{U \rho U^{\dagger}} U \rho^{1 / 2}\right\|_{2}^{2} \\
& =\left\|\rho^{1 / 2}\right\|_{2}^{2} \\
& =1 .
\end{aligned}
$$

So from the definition of the derivative, this means that

$$
\begin{aligned}
\lim _{\alpha \rightarrow 1} \Delta_{\alpha}(\rho, \sigma, \mathcal{N}) & =\lim _{\alpha \rightarrow 1} \frac{\log Q_{\alpha}(\rho, \sigma, \mathcal{N})-\log Q_{1}(\rho, \sigma, \mathcal{N})}{\alpha-1} \\
& =\left.\frac{d}{d \alpha}\left[\log Q_{\alpha}(\rho, \sigma, \mathcal{N})\right]\right|_{\alpha=1} \\
& =\left.\frac{1}{Q_{1}(\rho, \sigma, \mathcal{N})} \frac{d}{d \alpha}\left[Q_{\alpha}(\rho, \sigma, \mathcal{N})\right]\right|_{\alpha=1} \\
& =\left.\frac{d}{d \alpha}\left[Q_{\alpha}(\rho, \sigma, \mathcal{N})\right]\right|_{\alpha=1} .
\end{aligned}
$$

Let $\alpha^{\prime} \equiv \alpha-1$. Consider that

$$
Q_{\alpha}(\rho, \sigma, \mathcal{N})=\operatorname{Tr}\left\{\rho^{\alpha} \sigma^{-\alpha^{\prime} / 2} \mathcal{N}^{\dagger}\left(\mathcal{N}(\sigma)^{\alpha^{\prime} / 2} \mathcal{N}(\rho)^{-\alpha^{\prime}} \mathcal{N}(\sigma)^{\alpha^{\prime} / 2}\right) \sigma^{-\alpha^{\prime} / 2}\right\}
$$


Now we calculate $\frac{d}{d \alpha} Q_{\alpha}(\rho, \sigma, \mathcal{N})$ :

$$
\begin{aligned}
& \frac{d}{d \alpha} \operatorname{Tr}\left\{\rho^{\alpha} \sigma^{-\alpha^{\prime} / 2} \mathcal{N}^{\dagger}\left(\mathcal{N}(\sigma)^{\alpha^{\prime} / 2} \mathcal{N}(\rho)^{-\alpha^{\prime}} \mathcal{N}(\sigma)^{\alpha^{\prime} / 2}\right) \sigma^{-\alpha^{\prime} / 2}\right\} \\
& =\operatorname{Tr}\left\{\left[\frac{d}{d \alpha} \rho^{\alpha}\right] \sigma^{-\alpha^{\prime} / 2} \mathcal{N}^{\dagger}\left(\mathcal{N}(\sigma)^{\alpha^{\prime} / 2} \mathcal{N}(\rho)^{-\alpha^{\prime}} \mathcal{N}(\sigma)^{\alpha^{\prime} / 2}\right) \sigma^{-\alpha^{\prime} / 2}\right\} \\
& +\operatorname{Tr}\left\{\rho^{\alpha}\left[\frac{d}{d \alpha} \sigma^{-\alpha^{\prime} / 2}\right] \mathcal{N}^{\dagger}\left(\mathcal{N}(\sigma)^{\alpha^{\prime} / 2} \mathcal{N}(\rho)^{-\alpha^{\prime}} \mathcal{N}(\sigma)^{\alpha^{\prime} / 2}\right) \sigma^{-\alpha^{\prime} / 2}\right\} \\
& +\operatorname{Tr}\left\{\rho^{\alpha} \sigma^{-\alpha^{\prime} / 2} \mathcal{N}^{\dagger}\left(\left[\frac{d}{d \alpha} \mathcal{N}(\sigma)^{\alpha^{\prime} / 2}\right] \mathcal{N}(\rho)^{-\alpha^{\prime}} \mathcal{N}(\sigma)^{\alpha^{\prime} / 2}\right) \sigma^{-\alpha^{\prime} / 2}\right\} \\
& +\operatorname{Tr}\left\{\rho^{\alpha} \sigma^{-\alpha^{\prime} / 2} \mathcal{N}^{\dagger}\left(\mathcal{N}(\sigma)^{\alpha^{\prime} / 2}\left[\frac{d}{d \alpha} \mathcal{N}(\rho)^{-\alpha^{\prime}}\right] \mathcal{N}(\sigma)^{\alpha^{\prime} / 2}\right) \sigma^{-\alpha^{\prime} / 2}\right\} \\
& +\operatorname{Tr}\left\{\rho^{\alpha} \sigma^{-\alpha^{\prime} / 2} \mathcal{N}^{\dagger}\left(\mathcal{N}(\sigma)^{\alpha^{\prime} / 2} \mathcal{N}(\rho)^{-\alpha^{\prime}}\left[\frac{d}{d \alpha} \mathcal{N}(\sigma)^{\alpha^{\prime} / 2}\right]\right) \sigma^{-\alpha^{\prime} / 2}\right\} \\
& +\operatorname{Tr}\left\{\rho^{\alpha} \sigma^{-\alpha^{\prime} / 2} \mathcal{N}^{\dagger}\left(\mathcal{N}(\sigma)^{\alpha^{\prime} / 2} \mathcal{N}(\rho)^{-\alpha^{\prime}} \mathcal{N}(\sigma)^{\alpha^{\prime} / 2}\right)\left[\frac{d}{d \alpha} \sigma^{-\alpha^{\prime} / 2}\right]\right\} \\
& =\left[\operatorname{Tr}\left\{\rho^{\alpha}[\log \rho] \sigma^{-\alpha^{\prime} / 2} \mathcal{N}^{\dagger}\left(\mathcal{N}(\sigma)^{\alpha^{\prime} / 2} \mathcal{N}(\rho)^{-\alpha^{\prime}} \mathcal{N}(\sigma)^{\alpha^{\prime} / 2}\right) \sigma^{-\alpha^{\prime} / 2}\right\}\right. \\
& -\frac{1}{2} \operatorname{Tr}\left\{\rho[\log \sigma] \sigma^{-\alpha^{\prime} / 2} \mathcal{N}^{\dagger}\left(\mathcal{N}(\sigma)^{\alpha^{\prime} / 2} \mathcal{N}(\rho)^{-\alpha^{\prime}} \mathcal{N}(\sigma)^{\alpha^{\prime} / 2}\right) \sigma^{-\alpha^{\prime} / 2}\right\} \\
& +\frac{1}{2} \operatorname{Tr}\left\{\rho \sigma^{-\alpha^{\prime} / 2} \mathcal{N}^{\dagger}\left([\log \mathcal{N}(\sigma)] \mathcal{N}(\sigma)^{\alpha^{\prime} / 2} \mathcal{N}(\rho)^{-\alpha^{\prime}} \mathcal{N}(\sigma)^{\alpha^{\prime} / 2}\right) \sigma^{-\alpha^{\prime} / 2}\right\} \\
& -\operatorname{Tr}\left\{\rho \sigma^{-\alpha^{\prime} / 2} \mathcal{N}^{\dagger}\left(\mathcal{N}(\sigma)^{\alpha^{\prime} / 2}[\log \mathcal{N}(\rho)] \mathcal{N}(\rho)^{-\alpha^{\prime}} \mathcal{N}(\sigma)^{\alpha^{\prime} / 2}\right) \sigma^{-\alpha^{\prime} / 2}\right\} \\
& +\frac{1}{2} \operatorname{Tr}\left\{\rho \sigma^{-\alpha^{\prime} / 2} \mathcal{N}^{\dagger}\left(\mathcal{N}(\sigma)^{\alpha^{\prime} / 2} \mathcal{N}(\rho)^{-\alpha^{\prime}} \mathcal{N}(\sigma)^{\alpha^{\prime} / 2}[\log \mathcal{N}(\sigma)]\right) \sigma^{-\alpha^{\prime} / 2}\right\} \\
& \left.-\frac{1}{2} \operatorname{Tr}\left\{\rho \sigma^{-\alpha^{\prime} / 2} \mathcal{N}^{\dagger}\left(\mathcal{N}(\sigma)^{\alpha^{\prime} / 2} \mathcal{N}(\rho)^{-\alpha^{\prime}} \mathcal{N}(\sigma)^{\alpha^{\prime} / 2}\right) \sigma^{-\alpha^{\prime} / 2}[\log \sigma]\right\}\right] .
\end{aligned}
$$

Taking the limit as $\alpha \rightarrow 1$ gives

$$
\begin{aligned}
\left.\frac{d}{d \alpha} Q_{\alpha}(\rho, \sigma, \mathcal{N})\right|_{\alpha=1}= & \operatorname{Tr}\left\{\rho[\log \rho] \Pi_{\sigma} \mathcal{N}^{\dagger}\left(\Pi_{\mathcal{N}(\sigma)} \Pi_{\mathcal{N}(\rho)} \Pi_{\mathcal{N}(\sigma)}\right) \Pi_{\sigma}\right\} \\
& -\frac{1}{2} \operatorname{Tr}\left\{\rho[\log \sigma] \Pi_{\sigma} \mathcal{N}^{\dagger}\left(\Pi_{\mathcal{N}(\sigma)} \Pi_{\mathcal{N}(\rho)} \Pi_{\mathcal{N}(\sigma)}\right) \Pi_{\sigma}\right\} \\
+ & \frac{1}{2} \operatorname{Tr}\left\{\rho \Pi_{\sigma} \mathcal{N}^{\dagger}\left([\log \mathcal{N}(\sigma)] \Pi_{\mathcal{N}(\sigma)} \Pi_{\mathcal{N}(\rho)} \Pi_{\mathcal{N}(\sigma)}\right) \Pi_{\sigma}\right\} \\
& -\operatorname{Tr}\left\{\rho \Pi_{\sigma} \mathcal{N}^{\dagger}\left(\Pi_{\mathcal{N}(\sigma)}[\log \mathcal{N}(\rho)] \Pi_{\mathcal{N}(\rho)} \Pi_{\mathcal{N}(\sigma)}\right) \Pi_{\sigma}\right\} \\
+ & \frac{1}{2} \operatorname{Tr}\left\{\rho \Pi_{\sigma} \mathcal{N}^{\dagger}\left(\Pi_{\mathcal{N}(\sigma)} \Pi_{\mathcal{N}(\rho)} \Pi_{\mathcal{N}(\sigma)}[\log \mathcal{N}(\sigma)]\right) \Pi_{\sigma}\right\} \\
& -\frac{1}{2} \operatorname{Tr}\left\{\rho \Pi_{\sigma} \mathcal{N}^{\dagger}\left(\Pi_{\mathcal{N}(\sigma)} \Pi_{\mathcal{N}(\rho)} \Pi_{\mathcal{N}(\sigma)}\right)[\log \sigma] \Pi_{\sigma}\right\}
\end{aligned}
$$


We now simplify the first four terms and note that the last two are Hermitian conjugates of the second and third:

$$
\begin{array}{r}
\operatorname{Tr}\left\{\rho[\log \rho] \Pi_{\sigma} \mathcal{N}^{\dagger}\left(\Pi_{\mathcal{N}(\sigma)} \Pi_{\mathcal{N}(\rho)} \Pi_{\mathcal{N}(\sigma)}\right) \Pi_{\sigma}\right\}=\operatorname{Tr}\left\{\rho[\log \rho] \mathcal{N}^{\dagger}\left(\Pi_{\mathcal{N}(\rho)}\right)\right\} \\
=\operatorname{Tr}\left\{\mathcal{N}(\rho[\log \rho]) \Pi_{\mathcal{N}(\rho)}\right\}=\operatorname{Tr}\left\{U \rho[\log \rho] U^{\dagger}\left(\Pi_{\mathcal{N}(\rho)} \otimes I_{E}\right)\right\} \\
=\operatorname{Tr}\left\{\Pi_{U \rho U^{\dagger}} U \rho[\log \rho] U^{\dagger}\left(\Pi_{\mathcal{N}(\rho)} \otimes I_{E}\right)\right\}=\operatorname{Tr}\left\{\Pi_{U \rho U^{\dagger}} U \rho[\log \rho] U^{\dagger}\right\} \\
=\operatorname{Tr}\{\rho[\log \rho]\},
\end{array}
$$

$$
\begin{array}{r}
\operatorname{Tr}\left\{\rho[\log \sigma] \Pi_{\sigma} \mathcal{N}^{\dagger}\left(\Pi_{\mathcal{N}(\sigma)} \Pi_{\mathcal{N}(\rho)} \Pi_{\mathcal{N}(\sigma)}\right) \Pi_{\sigma}\right\}=\operatorname{Tr}\left\{\rho[\log \sigma] \mathcal{N}^{\dagger}\left(\Pi_{\mathcal{N}(\rho)}\right)\right\} \\
=\operatorname{Tr}\left\{\mathcal{N}(\rho[\log \sigma])\left(\Pi_{\mathcal{N}(\rho)}\right)\right\}=\operatorname{Tr}\left\{U \rho[\log \sigma] U^{\dagger}\left(\Pi_{\mathcal{N}(\rho)} \otimes I_{E}\right)\right\} \\
=\operatorname{Tr}\left\{\Pi_{U \rho U^{\dagger}} U \rho U^{\dagger} U[\log \sigma] U^{\dagger}\left(\Pi_{\mathcal{N}(\rho)} \otimes I_{E}\right)\right\}=\operatorname{Tr}\left\{U \rho U^{\dagger} U[\log \sigma] U^{\dagger}\right\} \\
=\operatorname{Tr}\{\rho[\log \sigma]\}
\end{array}
$$

$$
\begin{array}{r}
\operatorname{Tr}\left\{\rho \Pi_{\sigma} \mathcal{N}^{\dagger}\left([\log \mathcal{N}(\sigma)] \Pi_{\mathcal{N}(\sigma)} \Pi_{\mathcal{N}(\rho)} \Pi_{\mathcal{N}(\sigma)}\right) \Pi_{\sigma}\right\}=\operatorname{Tr}\left\{\rho \mathcal{N}^{\dagger}\left([\log \mathcal{N}(\sigma)] \Pi_{\mathcal{N}(\rho)}\right)\right\} \\
=\operatorname{Tr}\left\{\mathcal{N}(\rho)[\log \mathcal{N}(\sigma)] \Pi_{\mathcal{N}(\rho)}\right\}=\operatorname{Tr}\{\mathcal{N}(\rho)[\log \mathcal{N}(\sigma)]\}
\end{array}
$$

$$
\begin{aligned}
\operatorname{Tr}\left\{\rho \Pi_{\sigma} \mathcal{N}^{\dagger}\left(\Pi_{\mathcal{N}(\sigma)}[\log \mathcal{N}(\rho)] \Pi_{\mathcal{N}(\rho)} \Pi_{\mathcal{N}(\sigma)}\right) \Pi_{\sigma}\right\}=\operatorname{Tr}\left\{\rho \mathcal{N}^{\dagger}\left([\log \mathcal{N}(\rho)] \Pi_{\mathcal{N}(\rho)}\right)\right\} \\
=\operatorname{Tr}\left\{\mathcal{N}(\rho)\left([\log \mathcal{N}(\rho)] \Pi_{\mathcal{N}(\rho)}\right)\right\}=\operatorname{Tr}\{\mathcal{N}(\rho)[\log \mathcal{N}(\rho)]\}
\end{aligned}
$$

This then implies that the following equality holds

$$
\begin{aligned}
\left.\frac{d}{d \alpha} Q_{\alpha}(\rho, \sigma, \mathcal{N})\right|_{\alpha=1}=\operatorname{Tr}\{\rho[\log \rho]\}- & \operatorname{Tr}\{\rho[\log \sigma]\} \\
& +\operatorname{Tr}\{\mathcal{N}(\rho)[\log \mathcal{N}(\sigma)]\}-\operatorname{Tr}\{\mathcal{N}(\rho)[\log \mathcal{N}(\rho)]\}
\end{aligned}
$$

Putting together (A.15) and (A.24), we can then conclude the statement of the theorem.

\section{B Auxiliary lemmas and proofs}

Lemma 22 Let $\mathcal{A}$ and $\mathcal{T}$ be compact metric spaces, and let $f: \mathcal{A} \times \mathcal{T} \rightarrow \mathbb{R}$ be a continuous function. Then, $g, h: \mathcal{A} \rightarrow \mathbb{R}$, defined as $g(\alpha)=\max _{t \in \mathcal{T}} f(\alpha, t)$ and $h(\alpha)=\min _{t \in \mathcal{T}} f(\alpha, t)$ are continuous.

Proof. By the Heine-Cantor theorem, $f$ is uniformly continuous. Hence, for every $\varepsilon>0$, there exists a $\delta>0$ such that $\left|f(\alpha, t)-f\left(\alpha^{\prime}, t^{\prime}\right)\right|<\varepsilon$ whenever $D_{\mathcal{A}}\left(\alpha, \alpha^{\prime}\right)<\delta$ and $D_{\mathcal{T}}\left(t, t^{\prime}\right)<\delta$, where $D_{\mathcal{A}}$ and $D_{\mathcal{T}}$ are the distance functions on $\mathcal{A}$ and $\mathcal{T}$ respectively. Now, given $\alpha \in \mathcal{A}$, let $t$ be such that $g(\alpha)=f(\alpha, t)$. Then, for any $\alpha^{\prime} \in \mathcal{A}$ with $D_{\mathcal{A}}\left(\alpha, \alpha^{\prime}\right)<\delta$ we have that

$$
g(\alpha)=f(\alpha, t)<f\left(\alpha^{\prime}, t\right)+\varepsilon \leqslant \max _{t^{\prime} \in \mathcal{T}} f\left(\alpha^{\prime}, t^{\prime}\right)+\varepsilon=g\left(\alpha^{\prime}\right)+\varepsilon .
$$


By symmetry, we then have that $\left|g(\alpha)-g\left(\alpha^{\prime}\right)\right|<\varepsilon$, which proves the continuity of $g$. A similar argument establishes the continuity of $h$.

Proof of Theorem 11, Let $\rho, \sigma$, and $\mathcal{N}$ be as given in Definition 9, Let

$$
G(z)=\left(\mathcal{N}(\rho)^{-z / 2} \mathcal{N}(\sigma)^{z / 2} \otimes I_{E}\right) U \sigma^{-z / 2} \rho^{(1+z) / 2} .
$$

In the equation

$$
\frac{1}{p_{\theta}}=\frac{\theta}{p_{0}}+\frac{1-\theta}{p_{1}}
$$

choose $p_{0}=2$ and $p_{1}=2$, so that $p_{\theta}=2$. Recalling that

$$
M_{k}=\sup _{t \in \mathbb{R}}\|G(k+i t)\|_{p_{k}},
$$

for $k=0,1$, we find that

$$
\|G(\theta)\|_{p_{\theta}} \leq M_{0}^{1-\theta} M_{1}^{\theta}
$$

For our choices, we find that

$$
\begin{aligned}
M_{0}= & \sup _{t \in \mathbb{R}}\|G(i t)\|_{2} \\
= & \sup _{t \in \mathbb{R}}\left\|\left(\mathcal{N}(\rho)^{-i t / 2} \mathcal{N}(\sigma)^{i t / 2} \otimes I_{E}\right) U \sigma^{-i t / 2} \rho^{(1+i t) / 2}\right\|_{2} \\
& =\left\|\rho^{1 / 2}\right\|_{2}=1, \\
M_{1}= & \sup _{t \in \mathbb{R}}\|G(1+i t)\|_{2} \\
= & \sup _{t \in \mathbb{R}}\left\|\left(\mathcal{N}(\rho)^{-(1+i t) / 2} \mathcal{N}(\sigma)^{(1+i t) / 2} \otimes I_{E}\right) U \sigma^{-(1+i t) / 2} \rho^{(1+(1+i t)) / 2}\right\|_{2} \\
= & \sup _{t \in \mathbb{R}}\left\|\left(\mathcal{N}(\rho)^{-1 / 2} \mathcal{N}(\sigma)^{i t / 2} \mathcal{N}(\sigma)^{1 / 2} \otimes I_{E}\right) U \sigma^{-1 / 2} \sigma^{-i t / 2} \rho\right\|_{2} \\
= & {\left[\exp _{t \in \mathbb{R}} D_{2}\left(\rho \|\left(\mathcal{U}_{\sigma,-t} \circ \mathcal{P}_{\sigma, \mathcal{N}} \circ \mathcal{U}_{\mathcal{N}(\sigma), t}\right)(\mathcal{N}(\rho))\right)\right]^{1 / 2} . }
\end{aligned}
$$

Applying the three-line theorem gives

$$
\begin{aligned}
& \left\|\left(\mathcal{N}(\rho)^{-\theta / 2} \mathcal{N}(\sigma)^{\theta / 2} \otimes I_{E}\right) U \sigma^{-\theta / 2} \rho^{(1+\theta) / 2}\right\|_{2} \\
& \leq\left[\exp \sup _{t \in \mathbb{R}} D_{2}\left(\rho \|\left(\mathcal{U}_{\sigma,-t} \circ \mathcal{P}_{\sigma, \mathcal{N}} \circ \mathcal{U}_{\mathcal{N}(\sigma), t}\right)(\mathcal{N}(\rho))\right)\right]^{\theta / 2},
\end{aligned}
$$

and after a logarithm gives

$$
\frac{2}{\theta} \log \left\|\left(\mathcal{N}(\rho)^{-\theta / 2} \mathcal{N}(\sigma)^{\theta / 2} \otimes I_{E}\right) U \sigma^{-\theta / 2} \rho^{(1+\theta) / 2}\right\|_{2} \leq \sup _{t \in \mathbb{R}} D_{2}\left(\rho \|\left(\mathcal{U}_{\sigma,-t} \circ \mathcal{P}_{\sigma, \mathcal{N}} \circ \mathcal{U}_{\mathcal{N}(\sigma), t}\right)(\mathcal{N}(\rho))\right)
$$

Take the limit as $\theta \searrow 0$ to get

$$
D(\rho \| \sigma)-D(\mathcal{N}(\rho) \| \mathcal{N}(\sigma)) \leq \sup _{t \in \mathbb{R}} D_{2}\left(\rho \|\left(\mathcal{U}_{\sigma,-t} \circ \mathcal{P}_{\sigma, \mathcal{N}} \circ \mathcal{U}_{\mathcal{N}(\sigma), t}\right)(\mathcal{N}(\rho))\right)
$$


Now we prove the other inequality. Let $\rho, \sigma$, and $\mathcal{N}$ be as given in Definition 1 and such that $\operatorname{supp}(\rho) \subseteq \operatorname{supp}(\sigma)$. Take

$$
G(z)=\left(\mathcal{N}(\rho)^{z / 2} \mathcal{N}(\sigma)^{-z / 2} \otimes I_{E}\right) U \sigma^{z / 2} \rho^{(1-z) / 2} .
$$

Then $M_{0}=1$ again and

$$
\begin{aligned}
M_{1} & =\sup _{t \in \mathbb{R}}\|G(1+i t)\|_{2} \\
& =\sup _{t \in \mathbb{R}}\left\|\left(\mathcal{N}(\rho)^{(1+i t) / 2} \mathcal{N}(\sigma)^{-(1+i t) / 2} \otimes I_{E}\right) U \sigma^{(1+i t) / 2} \rho^{(1-(1+i t)) / 2}\right\|_{2} \\
& =\sup _{t \in \mathbb{R}}\left\|\left(\mathcal{N}(\rho)^{1 / 2} \mathcal{N}(\sigma)^{-i t / 2} \mathcal{N}(\sigma)^{-1 / 2} \otimes I_{E}\right) U \sigma^{1 / 2} \sigma^{i t / 2} \rho^{0}\right\|_{2} \\
& =\exp \left\{-\inf _{t \in \mathbb{R}} D_{0}\left(\rho \|\left(\mathcal{U}_{\sigma,-t} \circ \mathcal{P}_{\sigma, \mathcal{N}} \circ \mathcal{U}_{\mathcal{N}(\sigma), t}\right)(\mathcal{N}(\rho))\right)\right\}^{1 / 2} .
\end{aligned}
$$

Applying the three-line theorem gives

$$
\begin{aligned}
\|\left(\mathcal{N}(\rho)^{\theta / 2} \mathcal{N}(\sigma)^{-\theta / 2} \otimes I_{E}\right) & U \sigma^{\theta / 2} \rho^{(1-\theta) / 2} \|_{2} \\
\leq & {\left[\exp \left\{-\inf _{t \in \mathbb{R}} D_{0}\left(\rho \|\left(\mathcal{U}_{\sigma,-t} \circ \mathcal{P}_{\sigma, \mathcal{N}} \circ \mathcal{U}_{\mathcal{N}(\sigma), t}\right)(\mathcal{N}(\rho))\right)\right\}\right]^{\theta / 2}, }
\end{aligned}
$$

which after taking a logarithm gives

$$
\frac{2}{-\theta} \log \left\|\left(\mathcal{N}(\rho)^{\theta / 2} \mathcal{N}(\sigma)^{-\theta / 2} \otimes I_{E}\right) U \sigma^{\theta / 2} \rho^{(1-\theta) / 2}\right\|_{2} \geq \inf _{t \in \mathbb{R}} D_{0}\left(\rho \|\left(\mathcal{U}_{\sigma,-t} \circ \mathcal{P}_{\sigma, \mathcal{N}} \circ \mathcal{U}_{\mathcal{N}(\sigma), t}\right)(\mathcal{N}(\rho))\right)
$$

Take the limit as $\theta \searrow 0$ to get

$$
D(\rho \| \sigma)-D(\mathcal{N}(\rho) \| \mathcal{N}(\sigma)) \geq \inf _{t \in \mathbb{R}} D_{0}\left(\rho \|\left(\mathcal{U}_{\sigma,-t} \circ \mathcal{P}_{\sigma, \mathcal{N}} \circ \mathcal{U}_{\mathcal{N}(\sigma), t}\right)(\mathcal{N}(\rho))\right)
$$

\section{Taylor expansions}

Here we show the following limit:

$$
\lim _{\alpha \rightarrow 1} f\left(\alpha, V_{\mathcal{N}(\sigma)}, V_{\sigma}\right)=f\left(1, V_{\mathcal{N}(\sigma)}, V_{\sigma}\right),
$$

where $f\left(\alpha, V_{\mathcal{N}(\sigma)}, V_{\sigma}\right)$ is defined as

$$
f\left(\alpha, V_{\mathcal{N}(\sigma)}, V_{\sigma}\right)=\frac{1}{\alpha-1} \log \left\|\left([\mathcal{N}(\rho)]^{(1-\alpha) / 2} V_{\mathcal{N}(\sigma)}[\mathcal{N}(\sigma)]^{(\alpha-1) / 2} \otimes I_{E}\right) U \sigma^{(1-\alpha) / 2} V_{\sigma} \rho^{\alpha / 2}\right\|_{2}^{2}
$$

and $f\left(1, V_{\mathcal{N}(\sigma)}, V_{\sigma}\right)$ in (4.10). From the fact that

$$
\left.\log \left\|\left([\mathcal{N}(\rho)]^{(1-\alpha) / 2} V_{\mathcal{N}(\sigma)}[\mathcal{N}(\sigma)]^{(\alpha-1) / 2} \otimes I_{E}\right) U \sigma^{(1-\alpha) / 2} V_{\sigma} \rho^{\alpha / 2}\right\|_{2}^{2}\right|_{\alpha=1}=0
$$


we know (from the definition of derivative) that $\lim _{\alpha \rightarrow 1} f\left(\alpha, V_{\mathcal{N}(\sigma)}, V_{\sigma}\right)$ is equal to

$$
\begin{gathered}
\left.\frac{d}{d \alpha} \log \left\|\left([\mathcal{N}(\rho)]^{(1-\alpha) / 2} V_{\mathcal{N}(\sigma)}[\mathcal{N}(\sigma)]^{(\alpha-1) / 2} \otimes I_{E}\right) U \sigma^{(1-\alpha) / 2} V_{\sigma} \rho^{\alpha / 2}\right\|_{2}^{2}\right|_{\alpha=1} \\
=\left.\frac{d}{d \alpha}\left\|\left([\mathcal{N}(\rho)]^{(1-\alpha) / 2} V_{\mathcal{N}(\sigma)}[\mathcal{N}(\sigma)]^{(\alpha-1) / 2} \otimes I_{E}\right) U \sigma^{(1-\alpha) / 2} V_{\sigma} \rho^{\alpha / 2}\right\|_{2}^{2}\right|_{\alpha=1} .
\end{gathered}
$$

We evaluate the latter derivative by employing Taylor expansions. Substitute $\alpha=1+\gamma$, so that the quantity inside the derivative operation is equal to

$$
\left\|\left([\mathcal{N}(\rho)]^{-\gamma / 2} V_{\mathcal{N}(\sigma)}[\mathcal{N}(\sigma)]^{\gamma / 2} \otimes I_{E}\right) U \sigma^{-\gamma / 2} V_{\sigma} \rho^{(1+\gamma) / 2}\right\|_{2}^{2},
$$

which we can rewrite as

$$
\left\|\left(\left[V_{\mathcal{N}(\sigma)}^{\dagger} \mathcal{N}(\rho) V_{\mathcal{N}(\sigma)}\right]^{-\gamma / 2}[\mathcal{N}(\sigma)]^{\gamma / 2} \otimes I_{E}\right) U \sigma^{-\gamma / 2}\left[V_{\sigma} \rho V_{\sigma}^{\dagger}\right]^{(1+\gamma) / 2}\right\|_{2}^{2}
$$

due to the unitary invariance of the norm. Now we use that

$$
\begin{aligned}
{\left[V_{\sigma} \rho V_{\sigma}^{\dagger}\right]^{(1+\gamma) / 2} } & =\left[V_{\sigma} \rho V_{\sigma}^{\dagger}\right]^{1 / 2}+\frac{\gamma}{2}\left[V_{\sigma} \rho V_{\sigma}^{\dagger}\right]^{1 / 2} \log \left[V_{\sigma} \rho V_{\sigma}^{\dagger}\right]+O\left(\gamma^{2}\right), \\
\sigma^{-\gamma / 2} & =I-\frac{\gamma}{2} \log \sigma+O\left(\gamma^{2}\right) \\
{[\mathcal{N}(\sigma)]^{\gamma / 2} } & =I+\frac{\gamma}{2} \log [\mathcal{N}(\sigma)]+O\left(\gamma^{2}\right) \\
{\left[V_{\mathcal{N}(\sigma)}^{\dagger} \mathcal{N}(\rho) V_{\mathcal{N}(\sigma)}\right]^{-\gamma / 2} } & =I-\frac{\gamma}{2} \log \left[V_{\mathcal{N}(\sigma)}^{\dagger} \mathcal{N}(\rho) V_{\mathcal{N}(\sigma)}\right]+O\left(\gamma^{2}\right) .
\end{aligned}
$$

The above implies that

$$
\begin{aligned}
& {\left[V_{\mathcal{N}(\sigma)}^{\dagger} \mathcal{N}(\rho) V_{\mathcal{N}(\sigma)}\right]^{-\gamma / 2}[\mathcal{N}(\sigma)]^{\gamma / 2} U \sigma^{-\gamma / 2}\left[V_{\sigma} \rho V_{\sigma}^{\dagger}\right]^{(1+\gamma) / 2} } \\
&=\left(I-\frac{\gamma}{2} \log \left[V_{\mathcal{N}(\sigma)}^{\dagger} \mathcal{N}(\rho) V_{\mathcal{N}(\sigma)}\right]\right)\left(I+\frac{\gamma}{2} \log [\mathcal{N}(\sigma)]\right) \times \\
& U\left(I-\frac{\gamma}{2} \log \sigma\right)\left(\left[V_{\sigma} \rho V_{\sigma}^{\dagger}\right]^{1 / 2}+\frac{\gamma}{2}\left[V_{\sigma} \rho V_{\sigma}^{\dagger}\right]^{1 / 2} \log \left[V_{\sigma} \rho V_{\sigma}^{\dagger}\right]\right)+O\left(\gamma^{2}\right) .
\end{aligned}
$$

By working out the right-hand side above and neglecting terms of second order in $\gamma$ and higher, we find that

$$
\begin{aligned}
& {\left[V_{\mathcal{N}(\sigma)}^{\dagger} \mathcal{N}(\rho) V_{\mathcal{N}(\sigma)}\right]^{-\gamma / 2}[\mathcal{N}(\sigma)]^{\gamma / 2} U \sigma^{-\gamma / 2}\left[V_{\sigma} \rho V_{\sigma}^{\dagger}\right]^{(1+\gamma) / 2} } \\
&=U\left[V_{\sigma} \rho V_{\sigma}^{\dagger}\right]^{1 / 2}- \frac{\gamma}{2} \log \left[V_{\mathcal{N}(\sigma)}^{\dagger} \mathcal{N}(\rho) V_{\mathcal{N}(\sigma)}\right] U\left[V_{\sigma} \rho V_{\sigma}^{\dagger}\right]^{1 / 2}+\frac{\gamma}{2} \log [\mathcal{N}(\sigma)] U\left[V_{\sigma} \rho V_{\sigma}^{\dagger}\right]^{1 / 2} \\
&-\frac{\gamma}{2} U[\log \sigma]\left[V_{\sigma} \rho V_{\sigma}^{\dagger}\right]^{1 / 2}+\frac{\gamma}{2} U\left[V_{\sigma} \rho V_{\sigma}^{\dagger}\right]^{1 / 2} \log \left[V_{\sigma} \rho V_{\sigma}^{\dagger}\right]+O\left(\gamma^{2}\right) .
\end{aligned}
$$

The Hermitian conjugate is

$$
\begin{aligned}
{\left[V_{\sigma} \rho V_{\sigma}^{\dagger}\right]^{1 / 2} U^{\dagger}-} & \frac{\gamma}{2}\left[V_{\sigma} \rho V_{\sigma}^{\dagger}\right]^{1 / 2} U^{\dagger} \log \left[V_{\mathcal{N}(\sigma)}^{\dagger} \mathcal{N}(\rho) V_{\mathcal{N}(\sigma)}\right]+\frac{\gamma}{2}\left[V_{\sigma} \rho V_{\sigma}^{\dagger}\right]^{1 / 2} U^{\dagger} \log [\mathcal{N}(\sigma)] \\
& -\frac{\gamma}{2}\left[V_{\sigma} \rho V_{\sigma}^{\dagger}\right]^{1 / 2}[\log \sigma] U^{\dagger}+\frac{\gamma}{2}\left[\log \left[V_{\sigma} \rho V_{\sigma}^{\dagger}\right]\right]\left[V_{\sigma} \rho V_{\sigma}^{\dagger}\right]^{1 / 2} U^{\dagger}+O\left(\gamma^{2}\right)
\end{aligned}
$$


Combining (C.12) with its Hermitian conjugate and neglecting higher order terms gives

$$
\begin{aligned}
{\left[V_{\sigma} \rho V_{\sigma}^{\dagger}\right] } & -\gamma\left[V_{\sigma} \rho V_{\sigma}^{\dagger}\right]^{1 / 2} \mathcal{N}^{\dagger}\left(\log \left[V_{\mathcal{N}(\sigma)}^{\dagger} \mathcal{N}(\rho) V_{\mathcal{N}(\sigma)}\right]\right)\left[V_{\sigma} \rho V_{\sigma}^{\dagger}\right]^{1 / 2} \\
+\gamma\left[V_{\sigma} \rho V_{\sigma}^{\dagger}\right]^{1 / 2} & \mathcal{N}^{\dagger}(\log [\mathcal{N}(\sigma)])\left[V_{\sigma} \rho V_{\sigma}^{\dagger}\right]^{1 / 2}-\gamma\left[V_{\sigma} \rho V_{\sigma}^{\dagger}\right]^{1 / 2}[\log \sigma]\left[V_{\sigma} \rho V_{\sigma}^{\dagger}\right]^{1 / 2} \\
& +\frac{\gamma}{2}\left[V_{\sigma} \rho V_{\sigma}^{\dagger}\right] \log \left[V_{\sigma} \rho V_{\sigma}^{\dagger}\right]+\frac{\gamma}{2}\left(\log \left[V_{\sigma} \rho V_{\sigma}^{\dagger}\right]\right)\left[V_{\sigma} \rho V_{\sigma}^{\dagger}\right]+O\left(\gamma^{2}\right) .
\end{aligned}
$$

Taking a trace gives

$$
\begin{aligned}
\operatorname{Tr}\{\rho\}-\gamma \operatorname{Tr}\left\{\left[V_{\sigma} \rho V_{\sigma}^{\dagger}\right] \mathcal{N}^{\dagger}\left(\log \left[V_{\mathcal{N}(\sigma)}^{\dagger} \mathcal{N}(\rho) V_{\mathcal{N}(\sigma)}\right]\right)\right\} \\
+\gamma \operatorname{Tr}\left\{\left[V_{\sigma} \rho V_{\sigma}^{\dagger}\right] \mathcal{N}^{\dagger}(\log [\mathcal{N}(\sigma)])\right\}-\gamma \operatorname{Tr}\{\rho[\log \sigma]\}+\gamma \operatorname{Tr}\{\rho \log \rho\}+O\left(\gamma^{2}\right) .
\end{aligned}
$$

We can now finally use the above development to conclude that

$$
\begin{aligned}
& \left.\frac{d}{d \alpha}\left\|\left([\mathcal{N}(\rho)]^{(1-\alpha) / 2} V_{\mathcal{N}(\sigma)}[\mathcal{N}(\sigma)]^{(\alpha-1) / 2} \otimes I_{E}\right) U \sigma^{(1-\alpha) / 2} V_{\sigma} \rho^{\alpha / 2}\right\|_{2}^{2}\right|_{\alpha=1} \\
& =\left.\frac{d}{d \gamma}\left\|\left(\left[V_{\mathcal{N}(\sigma)}^{\dagger} \mathcal{N}(\rho) V_{\mathcal{N}(\sigma)}\right]^{-\gamma / 2}[\mathcal{N}(\sigma)]^{\gamma / 2} \otimes I_{E}\right) U \sigma^{-\gamma / 2}\left[V_{\sigma} \rho V_{\sigma}^{\dagger}\right]^{(1+\gamma) / 2}\right\|_{2}^{2}\right|_{\gamma=0} \\
& =\operatorname{Tr}\{\rho[\log \rho-\log \sigma]\}-\operatorname{Tr}\left\{\mathcal{N}\left(\left[V_{\sigma} \rho V_{\sigma}^{\dagger}\right]\right)\left[\log \left[V_{\mathcal{N}(\sigma)}^{\dagger} \mathcal{N}(\rho) V_{\mathcal{N}(\sigma)}\right]-\log [\mathcal{N}(\sigma)]\right]\right\} \\
& =f\left(1, V_{\mathcal{N}(\sigma)}, V_{\sigma}\right) .
\end{aligned}
$$

A similar development with Taylor expansions leads to the conclusion that (4.20) holds. However here one should employ the method outlined in the proof of [WWY14, Proposition 11].

\section{References}

[Bei13] Salman Beigi. Sandwiched Rényi divergence satisfies data processing inequality. Journal of Mathematical Physics, 54(12):122202, December 2013. arXiv:1306.5920.

[BK02] Howard Barnum and Emanuel Knill. Reversing quantum dynamics with near-optimal quantum and classical fidelity. Journal of Mathematical Physics, 43(5):2097-2106, May 2002. arXiv:quant-ph/0004088.

[BL76] J. Bergh and Jorgen Löfström. Interpolation Spaces. Springer-Verlag Berlin Heidelberg, 1976.

[BLW15] Mario Berta, Marius Lemm, and Mark M. Wilde. Monotonicity of quantum relative entropy and recoverability. Quantum Information and Computation, 15(15 \& 16):13331354, November 2015. arXiv:1412.4067.

[BSW15a] Mario Berta, Kaushik Seshadreesan, and Mark M. Wilde. Rényi generalizations of the conditional quantum mutual information. Journal of Mathematical Physics, 56(2):022205, February 2015. arXiv:1403.6102. 
[BSW15b] Mario Berta, Kaushik P. Seshadreesan, and Mark M. Wilde. Rényi generalizations of quantum information measures. Physical Review A, 91(2):022333, February 2015. arXiv:1502.07977.

[CBTW15] Patrick J. Coles, Mario Berta, Marco Tomamichel, and Stephanie Wehner. Entropic uncertainty relations and their applications. November 2015. arXiv:1511.04857.

[CMW14] Tom Cooney, Milan Mosonyi, and Mark M. Wilde. Strong converse exponents for a quantum channel discrimination problem and quantum-feedback-assisted communication. August 2014. arXiv:1408.3373.

[Csi95] Imre Csiszár. Generalized cutoff rates and Rényi's information measures. IEEE Transactions on Information Theory, 41(1):26-34, January 1995.

[CW04] Matthias Christandl and Andreas Winter. "Squashed entanglement": An additive entanglement measure. Journal of Mathematical Physics, 45(3):829-840, March 2004. arXiv:quant-ph/0308088.

[Dat09] Nilanjana Datta. Min- and max-relative entropies and a new entanglement monotone. IEEE Transactions on Information Theory, 55(6):2816-2826, June 2009. arXiv:0803.2770.

[DFW15] Frédéric Dupuis, Omar Fawzi, and Stephanie Wehner. Entanglement sampling and applications. IEEE Transactions on Information Theory, 61(2):1093-1112, February 2015. arXiv:1305.1316.

$\left[\mathrm{DKF}^{+} 12\right] \quad$ Frédéric Dupuis, Lea Kramer, Philippe Faist, Joseph M. Renes, and Renato Renner. Proceedings of the XVIIth International Congress on Mathematical Physics, chapter Generalized Entropies, pages 134-153. World Scientific, August 2012. arXiv:1211.3141.

[Dup15] Frederic Dupuis. Chain rules for quantum Rényi entropies. Journal of Mathematical Physics, 56(2):022203, February 2015. arXiv:1410.5455.

[DW15] Nilanjana Datta and Mark M. Wilde. Quantum Markov chains, sufficiency of quantum channels, and Rényi information measures. Journal of Physics A: Mathematical and Theoretical, 48(50):505301, November 2015. arXiv:1501.05636.

[FL13] Rupert L. Frank and Elliott H. Lieb. Monotonicity of a relative Rényi entropy. Journal of Mathematical Physics, 54(12):122201, December 2013. arXiv:1306.5358.

[FR15] Omar Fawzi and Renato Renner. Quantum conditional mutual information and approximate Markov chains. Communications in Mathematical Physics, 340(2):575-611, December 2015. arXiv:1410.0664.

[Hir52] Isidore Isaac Hirschman. A convexity theorem for certain groups of transformations. Journal d'Analyse Mathématique, 2(2):209-218, December 1952.

[JRS $\left.{ }^{+} 15\right] \quad$ Marius Junge, Renato Renner, David Sutter, Mark M. Wilde, and Andreas Winter. Universal recovery from a decrease of quantum relative entropy. September 2015. arXiv:1509.07127. 
[Lin75] Göran Lindblad. Completely positive maps and entropy inequalities. Communications in Mathematical Physics, 40(2):147-151, June 1975.

[LMW13] Noah Linden, Milán Mosonyi, and Andreas Winter. The structure of Rényi entropic inequalities. Proceedings of the Royal Society A: Mathematical, Physical and Engineering Science, 469(2158):20120737, October 2013. arXiv:1212.0248.

[LR73a] Elliott H. Lieb and Mary Beth Ruskai. A fundamental property of quantum-mechanical entropy. Physical Review Letters, 30(10):434-436, March 1973.

[LR73b] Elliott H. Lieb and Mary Beth Ruskai. Proof of the strong subadditivity of quantummechanical entropy. Journal of Mathematical Physics, 14(12):1938-1941, December 1973.

[LW14] Ke Li and Andreas Winter. Squashed entanglement, $k$-extendibility, quantum Markov chains, and recovery maps. October 2014. arXiv:1410.4184.

[MLDS $\left.{ }^{+} 13\right]$ Martin Müller-Lennert, Frédéric Dupuis, Oleg Szehr, Serge Fehr, and Marco Tomamichel. On quantum Rényi entropies: a new definition and some properties. Journal of Mathematical Physics, 54(12):122203, December 2013. arXiv:1306.3142.

[NC10] Michael A. Nielsen and Isaac L. Chuang. Quantum Computation and Quantum Information. Cambridge University Press, 2010. 10th anniversary edition.

[Pet86a] Dénes Petz. Quasi-entropies for finite quantum systems. Reports in Mathematical Physics, 23(1):57-65, February 1986.

[Pet86b] Dénes Petz. Sufficient subalgebras and the relative entropy of states of a von Neumann algebra. Communications in Mathematical Physics, 105(1):123-131, March 1986.

[Pet88] Dénes Petz. Sufficiency of channels over von Neumann algebras. Quarterly Journal of Mathematics, 39(1):97-108, 1988.

[Rén61] Alfréd Rényi. On measures of entropy and information. Proceedings of the 4th Berkeley Symposium on Mathematics, Statistics and Probability, 1:547-561, 1961. held at the Statistical Laboratory, University of California, 1960, edited by J. Neyman (University of California Press, Berkeley).

[Ren05] Renato Renner. Security of Quantum Key Distribution. PhD thesis, ETH Zürich, December 2005. arXiv:quant-ph/0512258.

[RS75] Michael Reed and Barry Simon. Methods of Modern Mathematical Physics II: Fourier Analysis, Self-Adjointness. Academic Press, 1975.

[RW04] Renato Renner and Stefan Wolf. Smooth Rényi entropy and applications. In Proceedings of the 2007 International Symposium on Information Theory, page 232, 2004.

[SBW15] Kaushik P. Seshadreesan, Mario Berta, and Mark M. Wilde. Rényi squashed entanglement, discord, and relative entropy differences. Journal of Physics A: Mathematical and Theoretical, 48(39):395303, September 2015. arXiv:1410.1443. 
[SFR15] David Sutter, Omar Fawzi, and Renato Renner. Universal recovery map for approximate Markov chains. April 2015. arXiv:1504.07251.

[Suz85] Masuo Suzuki. Transfer-matrix method and Monte Carlo simulation in quantum spin systems. Physical Review B, 31(5):2957, March 1985.

[Tom12] Marco Tomamichel. A Framework for Non-Asymptotic Quantum Information Theory. PhD thesis, ETH Zurich, 2012. arXiv:1203.2142.

[Tom15] Marco Tomamichel. Quantum Information Processing with Finite Resources Mathematical Foundations. SpringerBriefs in Mathematical Physics. Springer, 2015. arXiv:1504.00233.

[Uhl76] Armin Uhlmann. The "transition probability" in the state space of a *algebra. Reports on Mathematical Physics, 9(2):273-279, 1976.

[Uhl77] Armin Uhlmann. Relative entropy and the Wigner-Yanase-Dyson-Lieb concavity in an interpolation theory. Communications in Mathematical Physics, 54(1):21-32, 1977.

[Ume62] Hisaharu Umegaki. Conditional expectations in an operator algebra IV (entropy and information). Kodai Mathematical Seminar Reports, 14(2):59-85, 1962.

[vEH14] Tim van Erven and Peter Harremoes. Rényi divergence and Kullback-Leibler divergence. IEEE Transactions on Information Theory, 60(7):3797-3820, July 2014. arXiv:1206.2459.

[Wil13] Mark M. Wilde. Quantum Information Theory. Cambridge University Press, 2013. arXiv:1106.1445.

[Wil14] Mark M. Wilde. Multipartite quantum correlations and local recoverability. Proceedings of the Royal Society A, 471:20140941, March 2014. arXiv:1412.0333.

[Wil15] Mark M. Wilde. Recoverability in quantum information theory. Proceedings of the Royal Society A, 471(2182):20150338, October 2015. arXiv:1505.04661.

[WWY14] Mark M. Wilde, Andreas Winter, and Dong Yang. Strong converse for the classical capacity of entanglement-breaking and Hadamard channels via a sandwiched Rényi relative entropy. Communications in Mathematical Physics, 331(2):593-622, October 2014. arXiv:1306.1586.

[Zha14] Lin Zhang. A stronger monotonicity inequality of quantum relative entropy: A unifying approach via Rényi relative entropy. March 2014. arXiv:1403.5343v1. 